\title{
Graft-versus-host disease of the CNS is mediated by TNF upregulation in microglia
}

\begin{abstract}
Nimitha R. Mathew, ${ }^{1,2}$ Janaki M. Vinnakota, ${ }^{1,2,3}$ Petya Apostolova, ${ }^{1,2,4}$ Daniel Erny, ${ }^{4,5}$ Shaimaa Hamarsheh, ${ }^{1,2}$ Geoffroy Andrieux, ${ }^{6,7,8}$ Jung-Seok Kim, ${ }^{9}$ Kathrin Hanke, ${ }^{1,2}$ Tobias Goldmann, ${ }^{5}$ Louise Chappell-Maor, ${ }^{9}$ Nadia El-Khawanky, ${ }^{1,2}$ Gabriele Ihorst, ${ }^{1,2}$ Dominik Schmidt, ${ }^{1,2}$ Justus Duyster, ${ }^{1,2}$ Jürgen Finke, ${ }^{1,2}$ Thomas Blank, ${ }^{5}$ Melanie Boerries, ${ }^{6,7,8}$ Bruce R. Blazar, ${ }^{10}$ Steffen Jung, ${ }^{9}$ Marco Prinz,, 5,11,12 and Robert Zeiser ${ }^{1,2,11}$

'Department of Medicine I, Faculty of Medicine, Medical Center; ${ }^{2}$ Department of Hematology, Oncology and Stem Cell Transplantation, Faculty of Medicine, Medical Center; ${ }^{3}$ Faculty of Biology; ${ }^{4}$ Berta-OttensteinProgramme for Clinician Scientists, Faculty of Medicine, Medical Center; 5 Institute for Neuropathology, Faculty of Medicine; and ${ }^{6}$ Institute of Medical Bioinformatics and Systems Medicine (IBSM); Medical Center-University of Freiburg, Medical Faculty, University of Freiburg, Freiburg, Germany. ${ }^{7}$ Cerman Cancer Consortium (DKTK), Freiburg, Germany. ${ }^{8}$ Cerman Cancer Research Center (DKFZ), Heidelberg, Cermany. ${ }^{9}$ Department of Immunology, Weizmann Institute of Science, Rehovot, Israel. ${ }^{10}$ Division of Blood and Marrow Transplantation, Masonic Cancer Center and Department of Pediatrics, University of Minnesota, Minneapolis, Minnesota, USA. "'Centre for Integrative Biological Signalling Studies (CIBSS), Signalling Research Centres; and ${ }^{12}$ Center for Basics in NeuroModulation, Faculty of Medicine; Albert Ludwig University of Freiburg, Freiburg, Germany.
\end{abstract}

Acute graft-versus-host disease (CVHD) can affect the central nervous system (CNS). The role of microglia in CNS-CVHD remains undefined. In agreement with microglia activation, we found that profound morphological changes and MHC-II and CD80 upregulation occurred upon GVHD induction. RNA sequencing-based analysis of purified microglia obtained from mice with CNS-CVHD revealed TNF upregulation. Selective TNF gene deletion in microglia of $C \times 3 \mathrm{cr} 7^{\text {creeR }}$ Tnf ${ }^{f / /}$ mice reduced MHC-II expression and decreased CNS T cell infiltrates and VCAM- $1^{+}$endothelial cells. GVHD increased microglia TCF- $\beta$-activated kinase-1 (TAK1) activation and NF-kB/p38 MAPK signaling. Selective Tak1 deletion in microglia using Cx3cr ${ }^{\text {creER }}$ Tak $1^{f / f l}$ mice resulted in reduced TNF production and microglial MHC-II and improved neurocognitive activity. Pharmacological TAK1 inhibition reduced TNF production and MHC-II expression by microglia, Th1 and Th17 T cell infiltrates, and VCAM-1 ${ }^{+}$ endothelial cells and improved neurocognitive activity, without blocking graft-versus-leukemia effects. Consistent with these findings in mice, we observed increased activation and TNF production of microglia in the CNS of CVHD patients. In summary, we prove a role for microglia in CNS-CVHD, identify the TAK1/TNF/MHC-II axis as a mediator of CNS-CVHD, and provide a TAK1 inhibitor-based approach against GVHD-induced neurotoxicity.

\section{Introduction}

Acute graft-versus-host disease (GVHD) is a life-threatening complication after allogeneic hematopoietic cell transplantation (allo-HCT). About $50 \%$ of patients with severe acute GVHD fail to respond to corticosteroids, and steroid-refractory severe GVHD has a dismal prognosis with a 1-year survival rate of less than $20 \%$ (1). GVHD was classically considered to involve only the skin, the intestinal tract, and the liver, which was defined as the "tissue tropism of acute GVHD." However, there is increasing evidence that the effects of acute GVHD are not limited to the 3 classical target organs, but can also occur in the central nervous system (CNS). Animal studies showed that the transfer of allogeneic $\mathrm{T}$ cells caused CNS infiltration by effector memory $\mathrm{T}$ cells during GVHD and apoptosis in neurons, as well as reduced exploratory activity, spatial learning, and memory, in the recipi-

Authorship note: NRM and JMV are co-first authors.

Conflict of interest: The authors have declared that no conflict of interest exists. Copyright: (5) 2020, American Society for Clinical Investigation.

Submitted: May 28, 2019; Accepted: December 11, 2019; Published: February 17, 2020.

Reference information: / Clin Invest. 2020;130(3):1315-1329.

https://doi.org/10.1172/JCl130272. ent mice (2). Evidence for CNS-GVHD was not restricted solely to the murine model, as other investigators reported that CNS infiltration by $\mathrm{CD}^{+} \mathrm{T}$ cells was a key feature of GVHD in nonhuman primates (3). Conversely, treatment of primates with immunoprophylaxis after allo-HCT reduced the abundance of $\mathrm{T}$ cell infiltration into the brain (3). Consistent with findings in preclinical models, human brain analysis of female sex-mismatched bone marrow transplant recipients has identified cell infiltrates derived from $\mathrm{Y}$ chromosome ${ }^{+}$donors (4). In agreement with the occurrence of CNS-GVHD in patients, neurological deficits and MRI findings have been reported in patients developing GVHD (5).

The role of microglia for CNS inflammation during GVHD has remained unclear. Here we show, for the first time to our knowledge, that GVHD caused activation of microglia cells. Based on selective genetic ablation of TGF- $\beta$-activated kinase-1 (TAK1) or tumor necrosis factor (TNF) in microglia, we identify the TAK1/TNF/MHC-II axis as a central mediator of CNS inflammation. In a translational approach, we show that TAK1 inhibition reduces GVHD-induced neurotoxicity in mice, which provides a scientific rationale for testing this approach in a phase I trial in humans. 
A Untreated

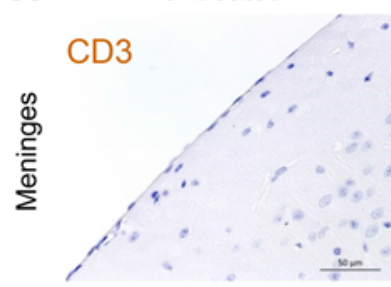

C



E

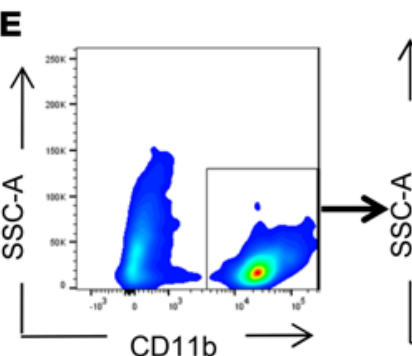

G

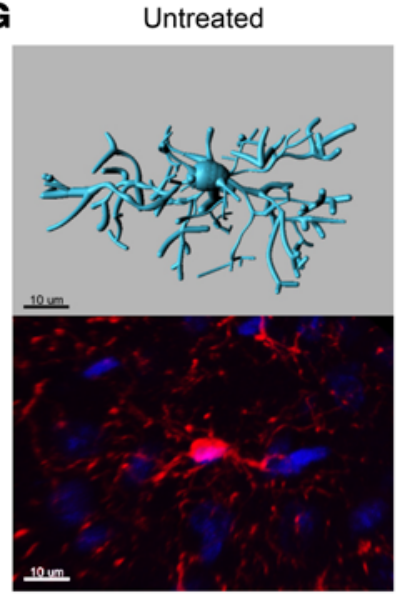

Syn-HCT

Allo-HCT
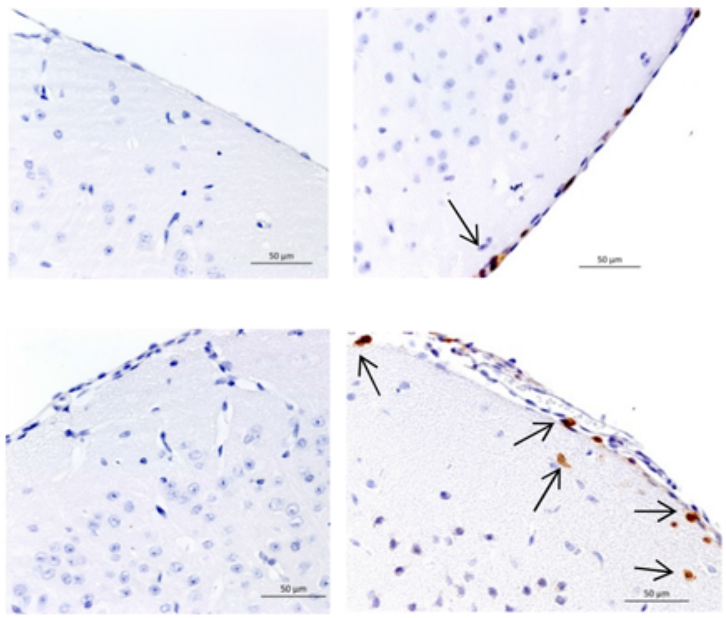

B

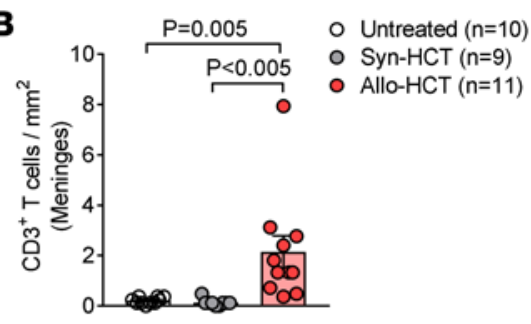

D

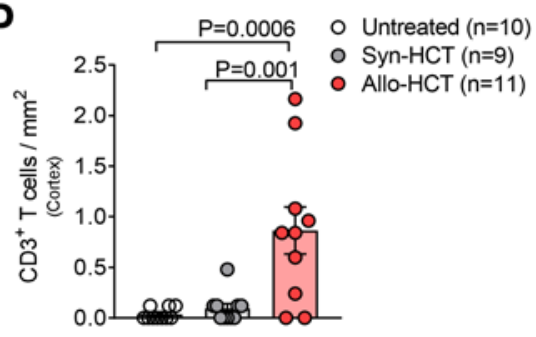

H

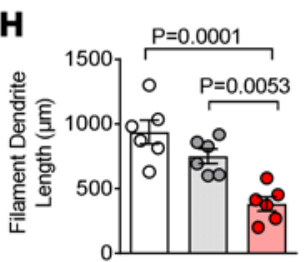

- Untreated $(n=6)$

- Syn-HCT $(n=6)$

- Allo-HCT $(n=6)$



- Untreated $(n=6)$

- Allo-HCT $(n=6)$
- Syn-HCT $(n=6)$

Syn-HCT
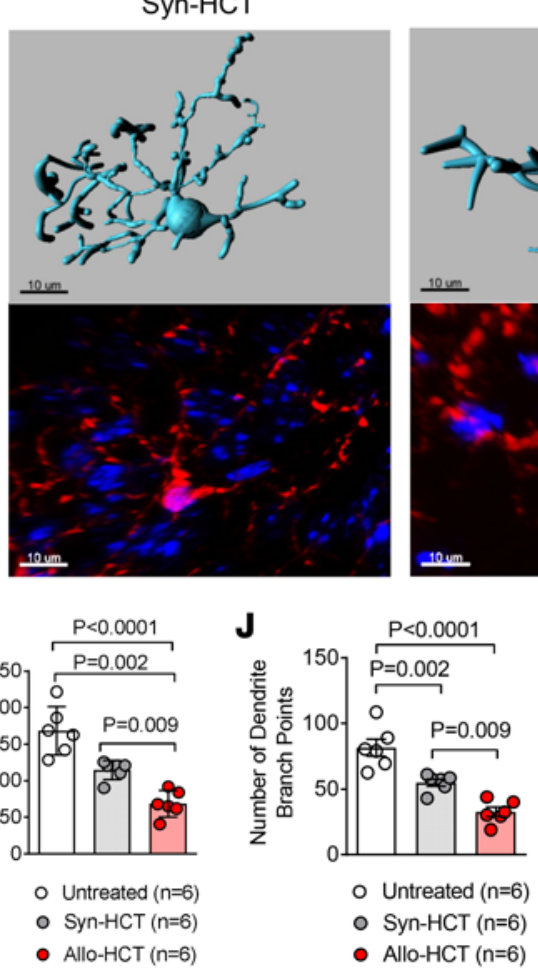

F

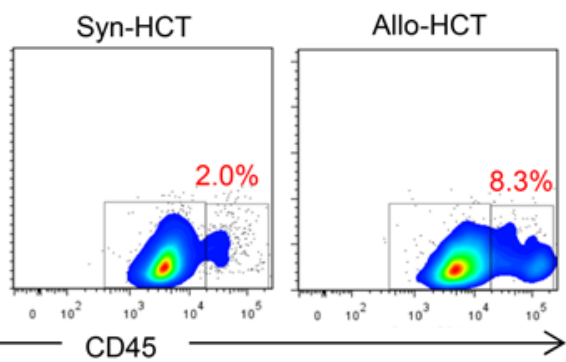

Allo-HCT




Figure 1. Microglia display activated morphology and T cells infiltrate the CNS during GVHD. (A-D) Histology of brain samples immunostained for $\mathrm{CD}^{+}$ T cells (brown) from untreated BALB/c mice $(n=10)$ or BALB/c mice on day 14 after syn-HCT $(n=9)$ or after allo-HCT $(n=11)$ as indicated. (A and C) A representative image from each group is shown. Scale bars: $50 \mu \mathrm{m}$. (B and $\mathbf{D}$ ) The scatter plots show the number of $\mathrm{CD}^{+} \mathrm{T}$ cells $\left(\right.$ per $\mathrm{mm}^{2}$ ) in cerebral meninges and cortex. The experiment was repeated 2 times, and the results (mean \pm SEM) were pooled. $P$ values were calculated using 1-way ANOVA. (E and $\mathbf{F}$ ) Flow cytometry for CD45 hi cells among CD11b+ cells in the CNS of untreated BALB/c mice $(n=10)$ or BALB/c mice on day 14 after syn-HCT $(n=10)$ or after allo-HCT $(n=11)$ as indicated. (E) A representative flow cytometry plot from each group is shown. (F) The scatter plot shows the quantification of CD45 hi cells among $\mathrm{CD}_{11 \mathrm{~b}^{+}}$cells from different groups as indicated. The experiment was repeated 3 times, and results (mean \pm SEM) were pooled. $P$ values were calculated using 1-way ANOVA. (C) Representative images showing Imarisbased (Bitplane) 3D reconstruction of $\mathrm{Iba}-1^{+}$microglia cells from untreated BALB/c mice or BALB/c mice on day 14 after syn-HCT or allo-HCT as indicated. Scale bar: $10 \mu \mathrm{m}$. (H-K) Scatter plots showing Imaris-based automated quantification of microglial morphology from microglia cells of untreated BALB/c mice $(n=6)$ or BALB/c mice on day 14 after syn-HCT $(n=6)$ or allo-HCT $(n=$ 6 ) as indicated. The experiment was repeated 2 times, and results (mean \pm SEM) were pooled. $P$ values were calculated using 1-way ANOVA.

\section{Results}

Microglia activation and expansion are features of CNS-GVHD. To determine whether GVHD induction in our models caused T cell infiltration into the CNS as a characteristic feature of CNS-GVHD (2), we analyzed the brains of mice that underwent syngeneic HCT (syn-HCT) or allogeneic HCT (allo-HCT). The abundance of $\mathrm{CD}^{+} \mathrm{T}$ cells increased in the cortex and the meninges of mice that underwent allo-HCT, but not syn-HCT (Figure 1, A-D). Allo$\mathrm{HCT}$ also led to an increased frequency of $\mathrm{CD} 11 \mathrm{~b}^{+} \mathrm{CD} 45^{\text {hi }}$ cells (Figure 1, E and F). The CD $11 \mathrm{~b}^{+} \mathrm{CD} 45^{\mathrm{hi}}$ cells in the CNS of mice undergoing allo-HCT included dendritic cells, macrophages, and monocytes (Supplemental Figure 1A; supplemental material available online with this article; https://oi.org/10.1172/ JCI130272DS1). Of these myeloid cells, monocytes were most abundant with a median of $23 \%$ (range $3 \%-35 \%$ ) of the CD $11 b^{+}$ $\mathrm{CD} 45^{\text {hi }}$ cells. To quantify the contribution of donor-derived monocytes to the increased TNF production and MHC-II expression, we used donor mice lacking CCR2, which exhibit reduced monocyte migration. We observed no reduction of TNF production or MHCII expression in the group that had received the bone marrow graft from $C C R 2^{-/}$donors (Supplemental Figure 1, B and C).

Hence we next analyzed the morphology of microglia cells. We observed that the filament dendrite length and the numbers of dendrite segments, branching points, and dendrite terminal points declined in mice that developed GVHD compared with mice that underwent syn-HCT or untreated mice (Figure 1, G-K). Comparable morphological changes have been previously reported as features of microglia activation in autoimmune disease of the CNS (6). In aggregate these findings show that profound morphological changes indicative of microglia activation occur upon CNS-GVHD induction.

MHC class II and CD80 expression is increased on microglia cells of mice developing GVHD. The CNS of mice undergoing allo-HCT harbored increased numbers of Iba- $1^{+}$microglia cells on day 14 after allo-HCT compared with syn-HCT (Figure 2, A and B). Conversely, the microglia decreased on day 7 in both groups receiving total-body irradiation (Supplemental Figure 1D). To characterize the transcriptional profile of microglia under GVHD conditions, we next isolated microglia based on CD11b and CD45 $5^{\text {lo }}$ expression from mice undergoing allo-HCT versus syn-HCT. RNA-Seq analysis showed close clustering of individual samples belonging to 1 group (Figure 2C). Microglia isolated from mice developing GVHD displayed a strong upregulation of genes involved in antigen presentation, in comparison with untreated mice or mice that had undergone syn-HCT (Figure 2D). In line with the RNA-Seq results, the microglia cells $\left(\mathrm{CD} 11 \mathrm{~b}^{+} \mathrm{CD} 45^{\mathrm{lo}}\right)$ expressed higher protein levels of MHC-II and CD80 on their surface, which have both been shown to be activation and maturation markers of myeloid cells (Figure 2, E-H). We also observed reduced expression of CX3CR1 on microglia upon GVHD induction (Figure 2, I and J) which is consistent with reports showing that this chemokine receptor declines on microglia upon activation (7).

Microglia cells exhibit increased TNF production upon CNSGVHD, and genetic deletion of TNF in microglia reduces disease activity. To further characterize the transcriptome of the activated microglia cells under GVHD conditions, we next analyzed their cytokine expression in mice undergoing allo-HCT versus synHCT. RNA-Seq analysis showed that multiple proinflammatory cytokines increased in microglia cells from the allo-HCT group (Figure 3A). A major GVHD-related cytokine that can mediate local cytotoxic effects is TNF. We hypothesized that TNF could be responsible for local tissue damage in the CNS because of its cytotoxic activity. To validate the findings on the protein levels, we next analyzed microglia by flow cytometry and found increased TNF production in mice that underwent allo-HCT compared with the syn-HCT group (Figure 3, B and C).

To probe whether microglial TNF plays a functional role in CNS-GVHD, we next induced GVHD in Cx3cr1 ${ }^{\text {creER }} \mathrm{Tnfl}^{\mathrm{fl}-\text { mice, }}$ which lack TNF in microglia. The $C x 3 c r 1^{\text {creER }}$ model was chosen because, following tamoxifen-induced gene deletion, short-living peripheral $\mathrm{CX} 3 \mathrm{CR} 1^{+}$monocytes and DCs are rapidly replenished within 4 weeks of tamoxifen induction and hence re-express the target gene, while in contrast the long-living microglia cells maintain the gene deletion (6). We found that $C \times 3 \mathrm{cr}^{\mathrm{creER}} \mathrm{Tnf} \mathrm{fl}^{\mathrm{l}-}$ mice exhibited lower frequencies of $\mathrm{CD}^{+}$cells in the cortex and meninges compared with littermate controls (Figure 3, D-F). RNA-Seq of microglia showed a reduction of Tnf, confirming the gene ablation, as well as of Cd74 and MHC-II (H2-Eb1) in Cx3 $\mathrm{crl}^{\text {creER }} \mathrm{Tnfl} /$ mice compared with littermate controls (Figure $3 \mathrm{G}$ ).

These studies identify TNF as a major pathogenic cytokine in CNS-GVHD based on unbiased RNA-Seq of microglia and functional gene deletion using the Cre-lox system.

GVHD enhances proinflammatory MAPK/NF- $\kappa B / T A K 1$ signaling in microglia cells. Since TNF, IL-6, IL-12, IL-18, and IL-1 $\beta$, which we had found in the RNA-Seq analysis of the microglia, activate various signaling cascades in immune cells, we next aimed at understanding which signaling events were responsible for the observed microglia activation. Transcriptome analysis of microglia showed upregulation of multiple signaling molecules, including $M a p 3 k 7$, encoding TGF- $\beta$-activated kinase-1 (TAK1) (Figure 4A). In agreement with this upregulation, we found increased phosphorylation of 38 MAPK, indicating the activation of the MAPK/NF- $\mathrm{BB}$ signaling pathway, in mice developing CNS-GVHD (Figure 4, B and C). 
A

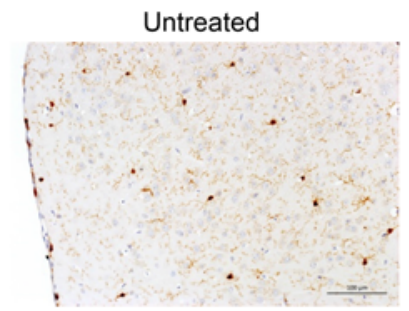

Iba-1

B

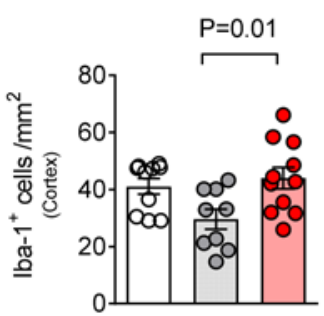

O Untreated $(n=10)$

- Syn-HCT $(n=9)$

- Allo-HCT $(n=11)$

E

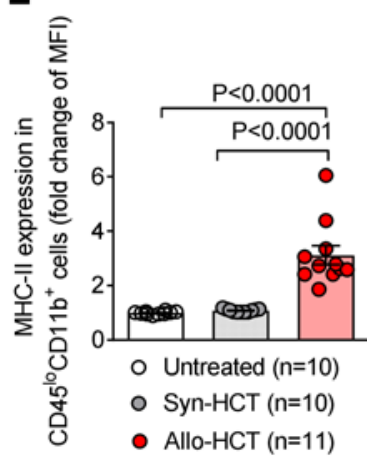

$\mathbf{F}$

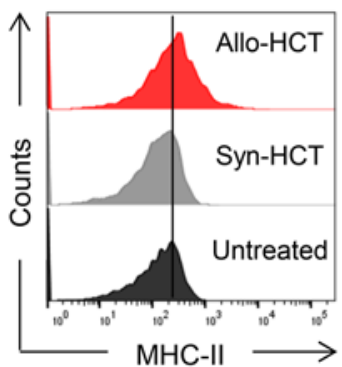

Syn-HCT



C

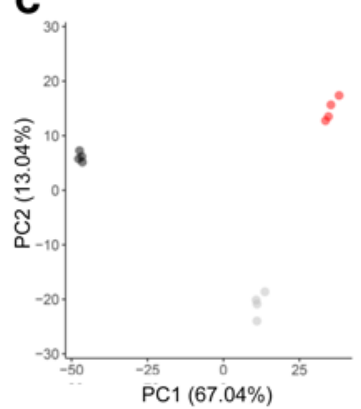

- Untreated $(n=4)$

Syn-HCT $(n=4)$

- Allo-HCT $(n=4)$

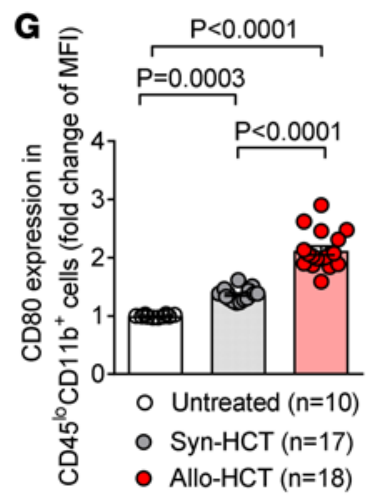

H



D

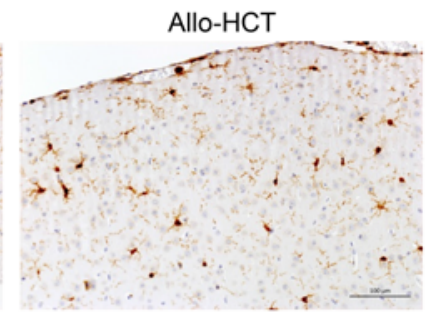

Untreated Syn-HCTAllo-HCT
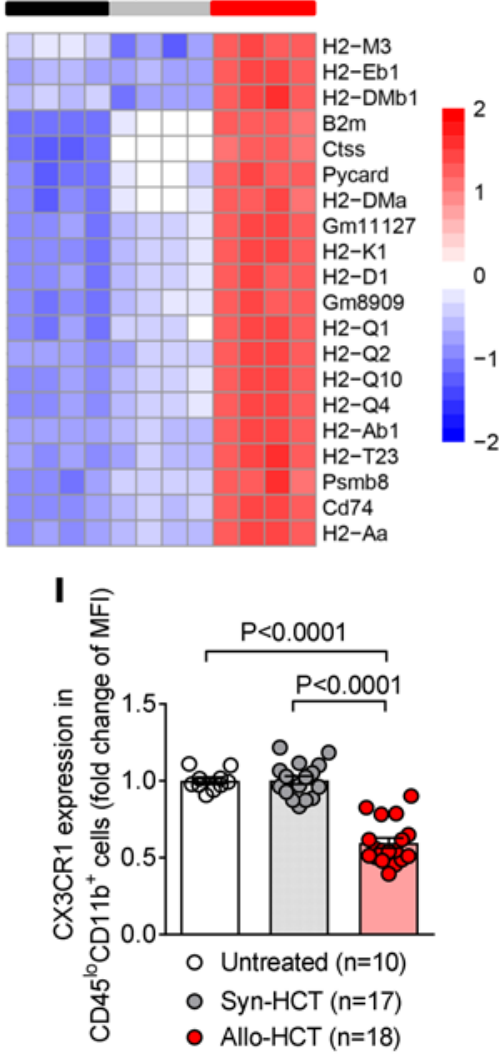

J

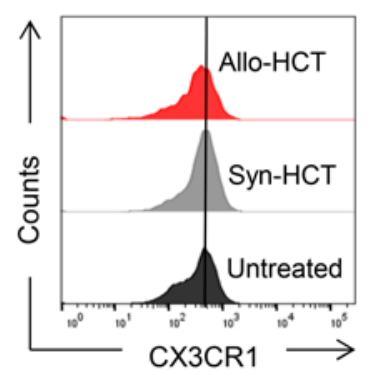

Figure 2. Microglial numbers and costimulatory molecules are increased during GVHD. (A) Histology of brain samples immunostained for lba-1+ $1^{+}$cells from untreated BALB/c mice or BALB/c mice on day 14 after syn-HCT or allo-HCT as indicated. Scale bars: $100 \mu \mathrm{m}$. (B) The scatter plot shows the number of Iba- $1^{+}$ cells (per $\left.\mathrm{mm}^{2}\right)$ in cerebral cortex from untreated BALB/c mice $(n=10)$ or BALB/c mice on day 14 after syn-HCT $(n=9)$ or allo -HCT $(n=11)$ as indicated. The experiment was repeated 2 times, and the results (mean \pm SEM) were pooled. $P$ values were calculated using 1 -way ANOVA. (C) Principal component (PC) analysis of RNA-Seq analysis of sorted microglia cells isolated from the CNS of untreated BALB/c mice $(n=4)$ or BALB/c mice on day 14 after syn-HCT $(n=4)$ or allo-HCT $(n=4)$. (D) Heatmap based on RNA-Seq showing the top 20 genes involved in antigen processing and presentation from microglia of untreated BALB/c mice $(n=4)$ or BALB/c mice on day 14 after syn-HCT $(n=4)$ or allo-HCT $(n=4)$. Color code represents the $Z$ score log intensity. $(E$ and $\mathbf{F})$ Scatter plot and respective flow cytometry plot showing quantification (fold change of MFI) of MHC-II expression on microglia (CD45 ${ }^{\circ} \mathrm{CD} 11 \mathrm{~b}^{+}$) from brains of untreated BALB/c mice $(n=10)$ or BALB/c mice on day 14 after syn-HCT $(n=10)$ or allo-HCT $(n=11)$ as indicated. (G-J) Scatter plot and respective flow cytometry plot showing quantification (fold change of MFI) of CD80 ( $\mathbf{G}$ and $\mathbf{H})$ and CX3CR1 (I and J) expression on microglia (CD45 $\left.5^{\circ} \mathrm{CD} 11 \mathrm{~b}^{+}\right)$from brains of untreated BALB/c mice $(n=10)$ or BALB/c mice on day 14 after syn-HCT $(n=17)$ or allo-HCT $(n=18)$ as indicated. The experiment was repeated 3 times, and results (mean \pm SEM) were pooled. $P$ values were calculated using 1-way ANOVA. 
A

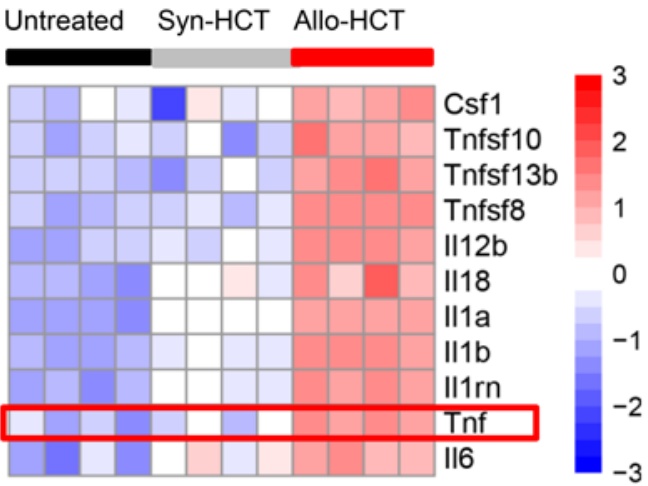

B

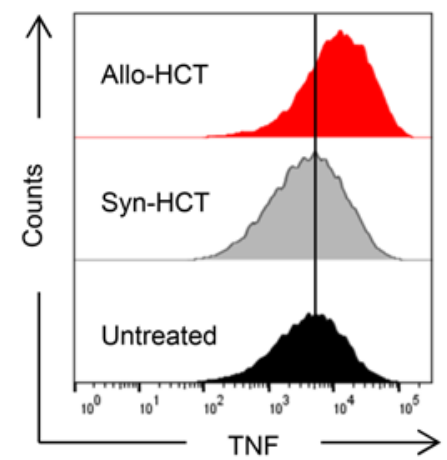

C



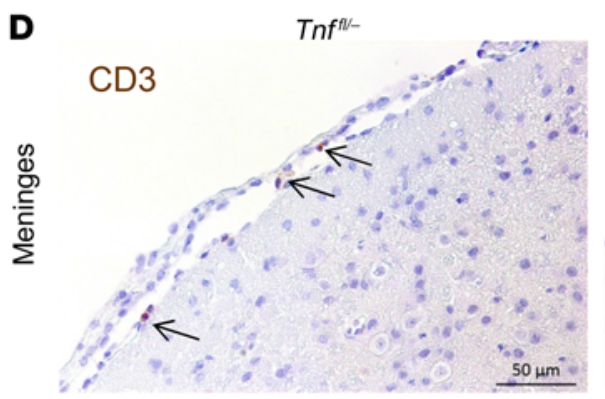

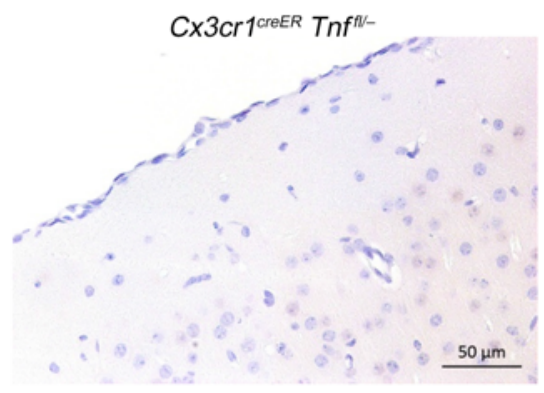

E

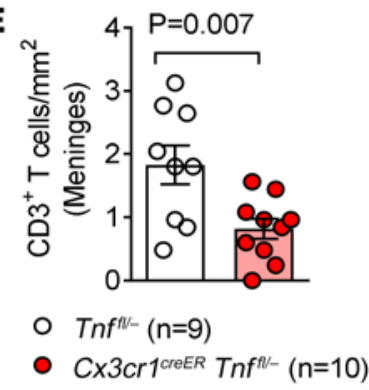

$\mathbf{F}$

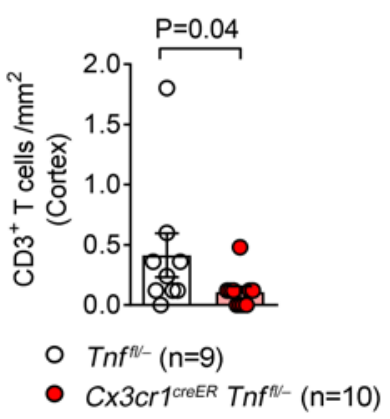

G



Figure 3. Microglia-derived TNF is essential for the infiltration of T cells into the brain. (A) Heatmap based on RNA-Seq showing the top 20 differentially regulated cytokines from microglia of untreated BALB/c mice $(n=4)$ or BALB/c mice on day 14 after syn-HCT $(n=4)$ or allo-HCT $(n=4)$. The color code represents the $Z$ score $\log _{2}$ intensity. (B) A representative flow cytometry plot showing intracellular TNF expression in microglia (CD45 ${ }^{\circ} \mathrm{CD} 11 \mathrm{~b}^{+}$) from brains of untreated BALB/c mice or BALB/c mice on day 14 after allo-HCT or syn-HCT. (C) The scatter plot shows the quantification (fold change of MFI) of intracellular TNF expression in microglia from the CNS of untreated BALB/C mice $(n=13)$ or BALB/c mice on day 14 after syn-HCT ( $n=18)$ or allo-HCT $(n=18)$ as indicated. The experiment was repeated 3 times, and the results (mean \pm SEM) were pooled. $P$ values were calculated using 1-way ANOVA. (D-F) Histology of brain samples for $\mathrm{CD}^{+} T$ cells from $\operatorname{Tn} f^{f / /}(n=9)$ and $C \times 3 \mathrm{cr}^{\mathrm{creER}} \mathrm{Tnf}^{f / /-}(n=10)$ mice on day 14 after allo-HCT as indicated. (D) Representative images showing meningeal $\mathrm{CD}^{+} \mathrm{T}$ cells in each group. Scale bars: $50 \mu \mathrm{m}$. (E and $\mathbf{F}$ ) The scatter plots show the number of $\mathrm{CD}^{+} \mathrm{T}$ cells $\left(\right.$ per $\left.\mathrm{mm} \mathrm{m}^{2}\right)$ in cerebral meninges (E) and cortex $(\mathbf{F})$ of $T n f^{f / /-}(n=9)$ and $\left[x 3 c r 7^{c r e E R} T n f^{f / /-}(n=10)\right.$ mice. The experiment was performed once. P values were calculated using 2-sided Student's unpaired $t$ test $(\mathbf{E})$ and 2-sided Mann-Whitney $U$ test (F). (G) Volcano plot based on RNA-Seq showing the top differentially regulated genes in $\operatorname{Tnf} f^{f /-}(n=9)$ and $C \times 3 \mathrm{cr}^{\text {creeR }} \mathrm{Tnf}^{\mathrm{fl} /-}(n=10)$ mice on day 14 after allo-HCT as indicated. Cd74, Tnf, and H2-Eb are upregulated in microglia of the Tnf fl/- mice compared with the $C \times 3 c r 7^{\text {creeR }}$ Tnf $f^{f /-}$ mice.

This finding is consistent with our observation that TNF expression is increased in microglia and with published data showing that $\mathrm{TAK} 1 / \mathrm{NF}-\kappa \mathrm{B}$ signaling triggers TNF production (8).

TNF can be induced by NF- $\mathrm{BB}$ signaling but can also by itself amplify NF- $\mathrm{B}$ pathway activity. Therefore, we studied the effect of TNF on NF- $\mathrm{KB}$ signaling in microglia in vitro by exposing the cells to increasing TNF concentrations. We observed that TNF caused activation of TAK1, JNK, and p65 (Figure 4, D-I). In agreement with this result, I $\mathrm{B}$ was increasingly degraded, a sign of higher NF- $\kappa \mathrm{B}$ activity (Figure $4 \mathrm{~J}$ ). These findings show that the $\mathrm{TAK} 1 / \mathrm{NF}-\kappa \mathrm{B}$ signaling cascade is active in microglia and can be induced in vitro by TNF exposure. 
A
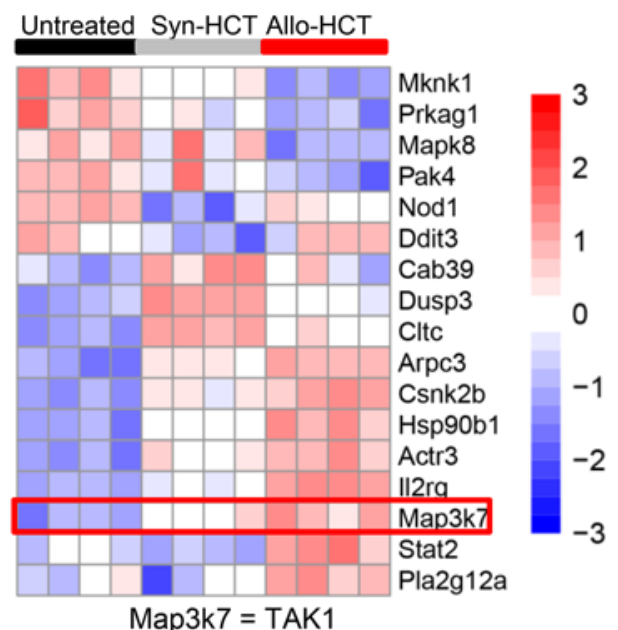

D



E



H

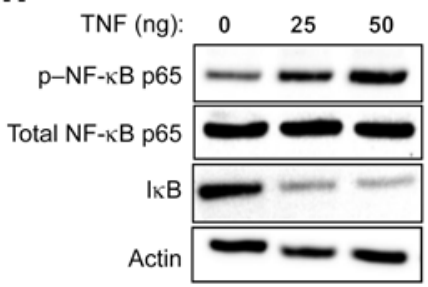

B

I

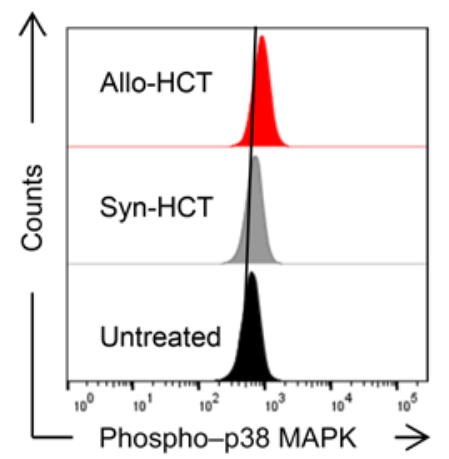

$\mathbf{F}$
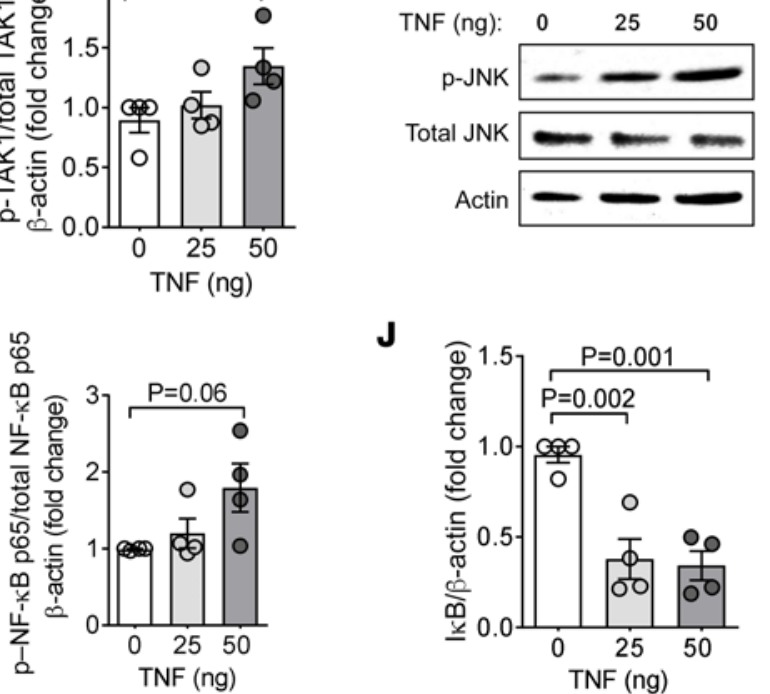

J

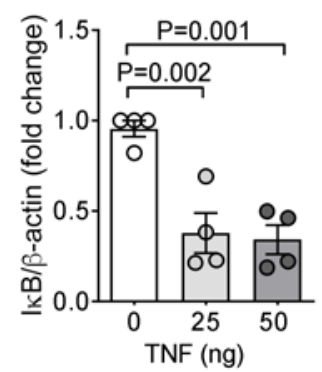

C


Figure 4. Downstream targets of TAK1 signaling are elevated in microglia during GVHD. (A) Heatmap based on RNA-Seq showing the top hits of PI3K/ Akt/mTOR signaling pathway from the microglia of untreated BALB/c mice $(n=4)$ or BALB/c mice on day 14 after allo-HCT $(n=4)$ or syn-HCT $(n=4)$. Color code represents the $Z$ score $\log _{2}$ intensity. (B) A representative flow cytometry plot showing intracellular phospho-p38 MAPK in microglia (CD45'o CD11b') from brains of untreated BALB/c mice or BALB/c mice on day 7 after syn-HCT or allo-HCT. (C) The scatter plot shows the quantification (fold change of MFI) of intracellular phospho-p38 MAPK expression in microglia from brains of untreated BALB/c mice $(n=14)$ or BALB/c mice on day 7 after syn-HCT ( $n=15)$ or allo-HCT $(n=15)$ as indicated. The experiment was repeated 3 times, and the results (mean \pm SEM) were pooled. The $P$ values were calculated using 1 -way ANOVA. (D-J) Western blot using protein derived from primary murine microglia treated with different concentrations of murine TNF for 24 hours as indicated. (D, F, and $\mathbf{H})$ Representative Western blot images showing the expression of phospho-TAK1 and total TAK1 (D), phospho-JNK and total JNK (F), and phos-



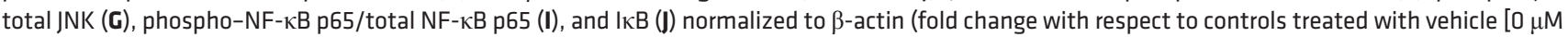
TNF]) in microglia treated as described. The experiment was repeated 4 times, and the results (mean \pm SEM) were pooled with $n=4$ biologically independent samples per group. Each data point represents an individual sample of 1 independent cell culture experiment. $P$ values were calculated using 1 -way ANOVA.

Deletion of TAK1 in microglia reduces CNS-GVHD. It was previously shown that upon stimulation TAK1 and its adaptors - TAB2, TAB3, and NEMO - are recruited to the polyubiquitinated receptor-interacting (TNFSF-interacting) serine-threonine kinase 1 (RIPK1), in turn allowing TAK1 to phosphorylate and activate the catalytic inhibitor of IKB kinase (IKK) subunits (9). The resulting IKK activation induces expression of multiple cytokines and chemokines. In this context, TAK1 was shown to be a central mediator in proinflammatory cytokine signaling, including TRAF6/TNF (10). Induction of GVHD in Cx3crr $1^{\text {creer }}$
$T a k 1^{f / f l}$ mice that lack TAK1 in microglia but not peripheral macrophages (Supplemental Figure 1, E-H) caused reduced $\mathrm{CD}^{+} \mathrm{T}$ cell frequencies in the meninges and cortex in comparison with littermate controls (Figure 5, A-D).

In line with reduced CNS inflammation, $C \times 3 \mathrm{cr} 1^{\text {creeER }} \mathrm{Tak} 1^{\text {It/fl }}$ mice exhibited lower numbers of $\mathrm{Iba}-1^{+}$cells in the meninges and cortex compared with littermate controls (Figure 5, E and F). Also lower MHC-II levels were found on microglia of CX3 $\mathrm{cr}^{\text {creer }} \mathrm{Tak1} 1^{\mathrm{f} / \mathrm{f}}$ mice compared with littermate controls (Figure $5, \mathrm{G}$ and $\mathrm{H}$ ). Consistent with a concept that TAK1 promotes 
TNF production, we observed lower TNF expression in microglia of $C \times 3 \mathrm{cr}^{\text {creER }} \mathrm{Tak} 1^{f l / f l}$ mice compared with littermate controls (Figure 5, I and J).

To analyze whether the CX3CR1-Cre system was specifically active after tamoxifen treatment, we used $C x 3 c r 1^{\text {creER }} T a k 1^{f / f l}$ mice without tamoxifen treatment. There was no evidence for TAK1 deletion in $\mathrm{C} \times 3 \mathrm{cr} 1^{\text {creER }} \mathrm{Tak} 1^{f / f l}$ mice without tamoxifen treatment (Supplemental Figure 1, I and J). Also, there was no evidence for Cre expression in neurons of $C \times 3 c r 1^{\text {creER }}$ R26-Tomato reporter mice (Supplemental Figure $1 \mathrm{~K}$ ). We observed no reduction of TNF or MHC-II in the $C \times 3 \mathrm{cr} 1^{\text {creER }} \mathrm{Tak}^{\mathrm{fl} / \mathrm{fl}}$ mice that were not treated with tamoxifen after allo-HCT in comparison with Tak1 $1^{f / f l}$ mice that underwent allo-HCT (Supplemental Figure 2, A and B).

The TAK1-deletion effect was restricted to the CNS, as GVHD-related mortality was not different when $\mathrm{Cx} 3 \mathrm{cr} 1^{\mathrm{creER}} \mathrm{Tak} 1^{\mathrm{fl} / \mathrm{fl}}$ mice and littermates were compared (Figure 5K). Consistent with similar survival rates, the histopathological scoring of peripheral GVHD organs including small intestines, large intestines, and liver was not different between $C x 3 c r 1^{\text {creER }} \mathrm{Tak}^{1 / / f l}$ mice and littermate controls (Supplemental Figure 2, C-E).

However, consistent with improved neurocognitive function, $C x 3 c r 1^{\text {creER }} \mathrm{Tak}^{\mathrm{fl} / \mathrm{fl}}$ mice exhibited higher exploratory activity in the elevated plus maze test compared with littermate controls (Figure $5 \mathrm{~L}$ ). Additionally, the novel object recognition test showed that $\mathrm{C} x 3 \mathrm{Cr}^{\mathrm{creER}} \mathrm{Tak1^{f/fl }}$ mice had a better recognition memory compared with controls (Figure $5 \mathrm{M}$ ). The differences in motoric function were not due to a general weakness of the mice, as the CX3cr $1^{\text {creER }}$ Tak $1^{f l / f l}$ mice and controls exhibited similar grip strength (Figure 5N). Also, the neurocognitive differences were not due to a loss of vision, as the $C x 3 c r 1^{\text {creeR }} T a k 1^{f l / f l}$ mice and controls exhibited similar results in the visual cliff test (Supplemental Figure $2 \mathrm{~F}$ ).

These findings indicate that a selective deletion of TAK1 in CX3CR1 ${ }^{+}$microglia reduces inflammatory $\mathrm{T}$ cell infiltration into the brain, reduces numbers of Iba- $1^{+}$microglia, reduces MHC-II and TNF expression by microglia, and promotes neurocognitive function of mice developing GVHD without blocking peripheral GVHD.

Therapeutic TAK1 inhibition reduces inflammatory features of microglia during CNS-GVHD. To test for a potentially translational approach, we next treated mice with 2 different TAK1 inhibitors, takinib and (5Z)-7-oxozeaenol, in comparison with vehicle and then analyzed the CNS. We observed that TAK1 inhibition reduced the expression of MHC-II and TNF compared with the vehicle treatment (Figure 6, A-D). Additionally, TAK1 inhibition reduced the number of $\mathrm{CD}^{+} \mathrm{T}$ cells and Iba- $1^{+}$microglia in comparison with the vehicle group (Figure 6, E-I).

In addition to the decreased accumulation of total $\mathrm{CD}^{+} \mathrm{T}$ cells in the CNS upon TAK1 inhibition, we observed a decrease of CD $4^{+} \mathrm{IFN}-\gamma^{+} \mathrm{T}$ cells (Th1 cells) and CD $4^{+} \mathrm{IL}-17^{+} \mathrm{T}$ cells (Th17 cells) in the CNS of mice undergoing allo-HCT upon TAK1 inhibition compared with the vehicle group (Figure 6, J-M). Other T cell subpopulations, including naive $\mathrm{T}$ cells, central memory $\mathrm{T}$ cells, effector memory $\mathrm{T}$ cells, and $\mathrm{CD} 4^{+} \mathrm{IL}-4^{+} \mathrm{T}$ cells (Th2 cells), were not different in the takinib group compared with the vehicle group (Supplemental Figure 3, A-D). We observed a nonsignificant trend toward an increase of $\mathrm{CD} 4^{+} \mathrm{FoxP} 3^{+} \mathrm{T}$ regulatory cells in the $\mathrm{CNS}$ of mice undergoing allo-HCT upon TAK1 inhibition compared with the vehicle group (Supplemental Figure 3E).
To understand why $\mathrm{T}$ cell infiltration was reduced in the CNS of mice that were treated with the TAK1 inhibitor or lacking TNF in $\mathrm{CX} 3 \mathrm{CR} 1^{+}$cells, we studied molecules that are required for T cell extravasation. $\mathrm{T}$ cell adhesion to endothelial cells is mediated via VCAM-1 and ICAM-1 (11), which then allows T cells to exit the vessel and enter the CNS.

We found reduced expression of VCAM-1 and ICAM-1 on endothelial cells $\left(\mathrm{CD}_{31} 1^{+} \mathrm{CD} 105^{+}\right)$in the CNS of mice treated with takinib compared with mice treated with vehicle (Figure 7, A-E, and Supplemental Figure 4). We also found a reduced number of VCAM-1 ${ }^{+}$endothelial cells in the CNS of Cx3 $\mathrm{cr}^{\text {creER }} \mathrm{Tnfl}^{\mathrm{fl}-}$ mice (TNF deletion) compared with Tnfl/- (no TNF deletion) mice (Figure 7, F and G). These findings support the concept that the TAK1/TNF axis promotes VCAM-1 expression on endothelial cells. Genetic deletion of TNF or TAK1 inhibition reduces VCAM1 expression during CNS-GVHD.

These findings in the CNS of TAK1 inhibitor-treated mice were connected to improved neurocognitive function, as the TAK1 inhibitor group exhibited more frequent entries into open arm in the elevated plus maze test compared with the vehicle group (Figure $7 \mathrm{H})$. The novel object recognition test showed that the TAK1 inhibitor-treated group performed better compared with the vehicletreated group (Figure 7I). The differences in motoric function were not due to a general weakness of the TAK1 inhibitor-treated mice, as they exhibited similar grip strength (Supplemental Figure $5 \mathrm{~A})$. Also, the neurocognitive differences were not due to a loss of vision, as the TAK1 inhibitor-treated mice and the vehicle-treated mice exhibited similar results in the visual cliff test (Supplemental Figure $5 \mathrm{~B}$ ). In order to be potentially applicable in a clinical setting, engraftment of the donor hematopoietic system is essential. We observed that the TAK1 inhibitor-treated mice and the vehicle-treated mice exhibited similar engraftment (Supplemental Figure 5C), while there was no change of GVHD scores in the small and large bowels but a reduced liver GVHD score in the TAK inhibitor-treated mice (Supplemental Figure 5, D-F). TAK1 inhibitor-treated animals exhibited a lower frequency of monocyte infiltrates or activated microglia compared with vehicle-treated mice (Supplemental Figure 5, G and H).

To evaluate the impact of takinib treatment on graft-versus-leukemia (GVL) activity, we used a genetic acute myeloid leukemia (AML) model (FLT3-ITD/MLL-PTD) and a cell line-based AML model (WEHI-3B) (12). The AML cells were on the same genetic background as the recipient mice to mimic the clinical situation. In both models the group that received $\mathrm{T}$ cells experienced improved survival compared with the group that received AML cells and bone marrow only, indicating the GVL effect. The treatment with takinib did not reduce the GVL effect in both leukemia models (Figure 7, J and K).

Microglia cells expand and produce TNF in human CNS-GVHD. To understand whether patients developing acute GVHD had changes in microglia cells comparable to those we had observed in mice, we studied the brains of patients who died after allo-HCT. We observed an increased number of Iba- $1^{+}$microglia in patients who developed acute GVHD compared with patients who underwent allo-HCT but did not develop GVHD. The increase was found in gray and white matter of the frontal lobe (Figure 8, A-D). TNF in microglia was visualized by IHC for Iba-1 and TNF. The 
A Tak1

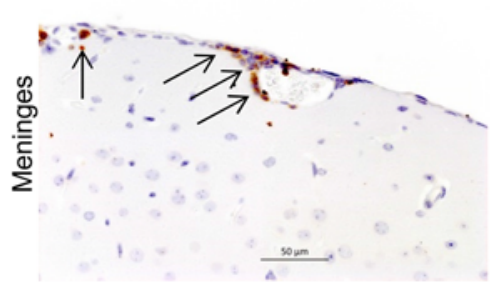

C

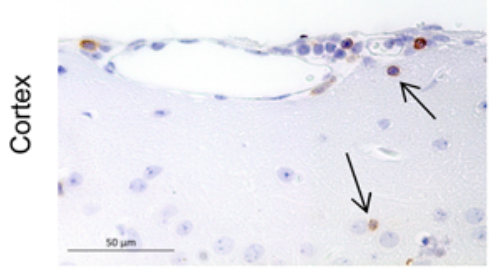

E

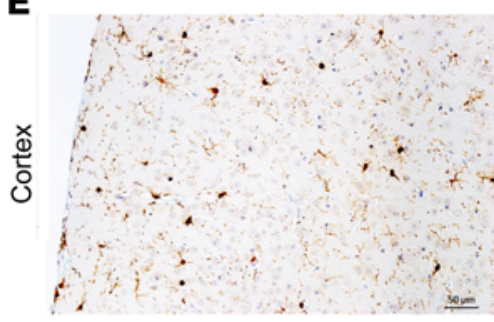

CX3cr1creER Tak1 1 finl

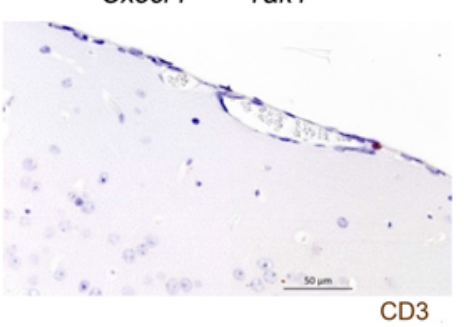

CD3

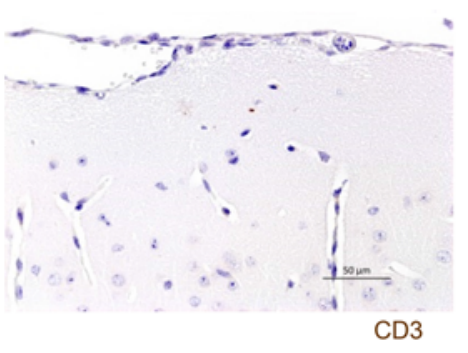

CD3

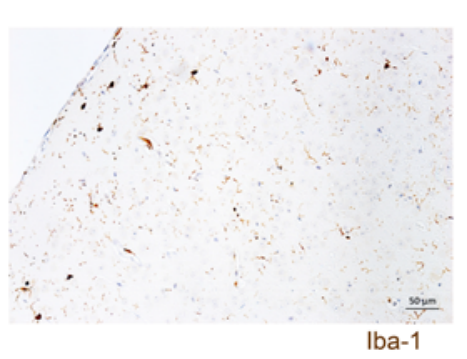

B

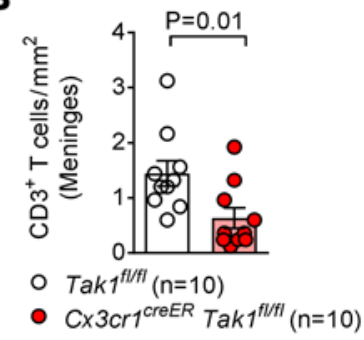

D

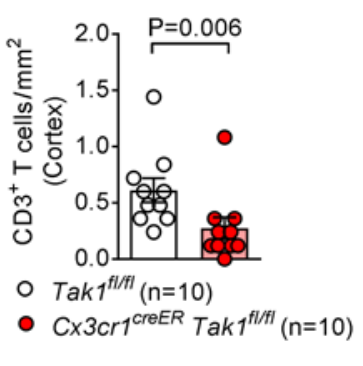

$F$

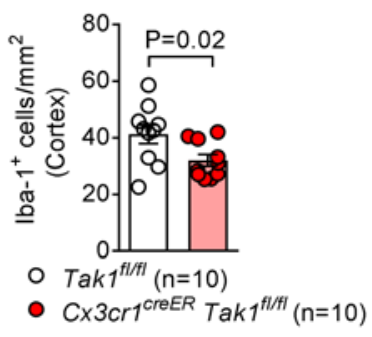

G



K

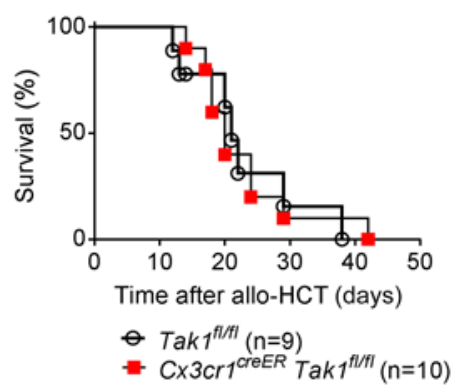

H



L Elevated plus maze test

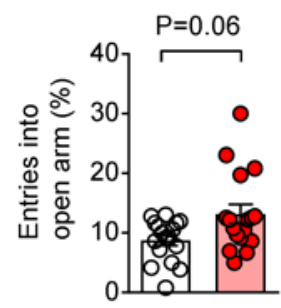

- Tak $1^{f l / f l}(\mathrm{n}=17)$

- $C \times 3{ }^{c r} 1^{\text {CreER }} \operatorname{Tak}^{f l f f l}(\mathrm{n}=17)$

I

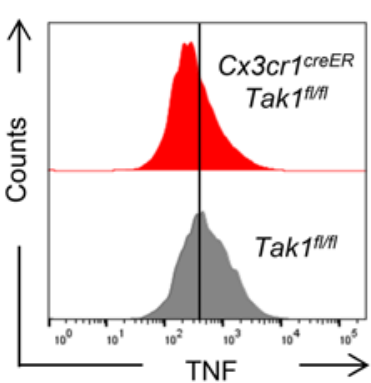

M Novel object recognition test

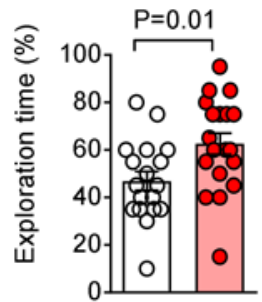

- $T a k 1^{f l / f l}(\mathrm{n}=18)$

- Cx3cr1 ${ }^{\text {CreER }}$ Tak $1^{f l i f l}(\mathrm{n}=19)$
J

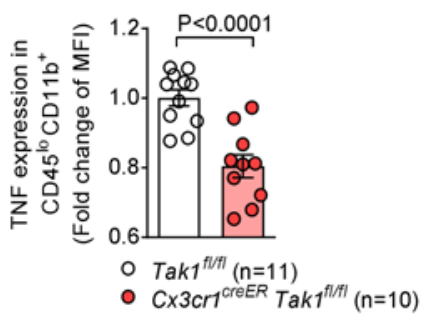

N Grip strength

test

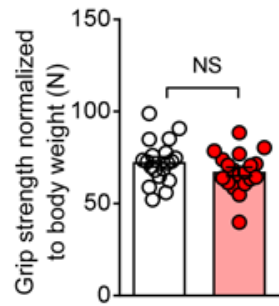

- $\operatorname{Tak1}^{f l / f I}(\mathrm{n}=19)$

- $C \times 3 c r 1^{\text {CreER }} \operatorname{Tak}^{f l / f l}(\mathrm{n}=19)$ 
Figure 5. Deficiency of TAK1 in microglia alleviates CNS-CVHD-associated pathology and cognitive and memory deficits. (A-F) Histology of brain samples immunostained for meningeal (A) and cortical (C) $\mathrm{CD}^{+} \mathrm{T}$ cells and cortical Iba- $1^{+}$cells (E) from Tak $7^{\text {fl/fl }}$ or $\left[\times 3 \mathrm{Cr}^{7 \text { reeER }} \operatorname{Tak}^{\text {fl/fl }}\right.$ ( $n=10$ each) mice on day 14 after allo-HCT. Scale bars: $50 \mu \mathrm{m}$. (B, D, and F) The scatter plots show the number of meningeal (B) and cortical (D) CD3 ${ }^{+} \mathrm{T}$ cells and cortical lba- $1^{+}$cells $(\mathbf{F})$. The experiment was repeated 3 times. The results (mean \pm SEM) were pooled. (G-J) Representative flow cytometry (G and I) and scatter ( $\mathbf{H}$ and $\mathbf{J})$ plots showing TNF and MHC-II expression in microglia

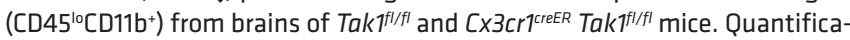
tion of MHC-II expression from Tak $7^{f l / f l}(n=10)$ and $C \times 3 c r$ creeR $^{\text {Tak }} 7^{f l / f l}(n=10)$

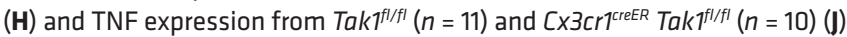
on day 14 after allo-HCT is shown. The experiment was repeated 3 times. The results (mean \pm SEM) were pooled. (K) Percentage survival of Tak $7^{f / f l}(n$ $=9)$ and $C \times 3 \mathrm{cr}^{\text {creeER }} \operatorname{Tak}^{f^{f / f l}(n=10)}$ mice that underwent allo-HCT. The experiment was performed 3 times. The results were pooled. (L) The scatter plot shows the percentage of open-arm entries by Tak $7^{f / f l}$ and $\left[\times 3 c r 7^{\text {creER }} T a k 7^{f / f f}\right.$ ( $n=17$ each) mice on day 21 after allo-HCT in an elevated plus maze test. (M) The scatter plot shows the percentage of time spent by Tak $7^{f / f l}(n=18)$ and $C \times 3 \mathrm{Cr}^{7 \mathrm{creER}} \operatorname{Tak} \mathrm{T}^{\mathrm{fl} / \mathrm{fl}}(n=19)$ mice in exploring a novel object with respect to the total time on day 19 after allo-HCT in a novel object recognition test (N) The scatter plot shows grip strength normalized to body weight $(\mathrm{N})$ of

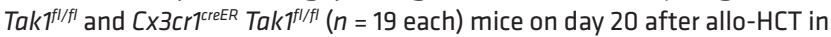
a grip strength test. The experiments were repeated 3 times. The results (mean $\pm \mathrm{SEM}$ ) were pooled. $P$ values were calculated using Mann-Whitney $U$ test (B, D, and $\mathbf{L}$ ), 2-sided Mantel-Cox test (K), or 2-sided Student's unpaired $t$ test $(\mathbf{F}, \mathbf{H}, \mathbf{J}, \mathbf{M}$, and $\mathbf{N})$.

frequency of TNF and Iba-1 double-positive microglia was significantly increased in patients that had developed acute GVHD (Figure 8, E and F). In agreement with CNS-GVHD, these patients also exhibited higher $\mathrm{CD}^{+} \mathrm{T}$ cell frequencies (Figure $8, \mathrm{G}$ and $\mathrm{H}$ ). These observations made in patient samples indicate that major findings made in the mouse model may also hold true for humans.

In addition, we reviewed the literature for cases of CNSGVHD and listed the reports in Supplemental Table 4. In the majority of the cases of CNS-GVHD the diagnosis was histology-proven, supporting the concept that CNS-GVHD is a clinically relevant complication.

To further elucidate the potential clinical relevance of CNSGVHD, we analyzed the frequency of neurological symptoms that occurred at the same time that a patient developed acute GVHD grade II-IV in 503 patients undergoing allo-HCT at Freiburg University Hospital, Germany. Neurological symptoms related to infection or vascular events were not included. The time interval included the first 2 years after allo-HCT because acute GVHD occurs in $90 \%$ of all cases within this time interval. The multivariable logistic regression analysis included presence of acute GVHD grade II-IV, donor type (MRD, MUD, MMUD, haploidentical donor), conditioning (myeloablative versus reduced-intensity), sex, and age over 40 versus under 40 years as possible risk factors for the occurrence of neurological complications. We found that development of acute GVHD grade II-IV was independently associated with increased risk of noninfectious neurological complications with an odds ratio of 491 and $P$ less than 0.0001 (Supplemental Tables 5 and 6). Our results are consistent with a recent retrospective analysis on the incidence and risk factors of noninfectious neurological complications in 971 consecutive patients with hematological malignancies undergoing allo-HCT (13). The authors found in multivariable analysis that development of acute
GVHD grade II-IV was independently associated with increased risk of noninfectious neurological complications with a hazard ratio of 3.3 and $P$ less than 0.00001 .

Despite these reports, it is important that other diseases such as progressive multifocal leukoencephalopathy or viral encephalitis (e.g., herpes simplex virus, CMV, or varicella zoster virus) be ruled out before CNS-GVHD is diagnosed in a patient with GVHD developing neurological symptoms.

\section{Discussion}

The clinical picture of acute GVHD is often connected to neurological deficits in patients, morphological CNS white matter changes detectable by MRI, and intraparenchymal lymphocytic infiltration of the brain upon autopsy $(5,14)$.

While previous work had shown that $\mathrm{T}$ cells contribute to CNS-GVHD $(2,3)$, the role of microglia cells had not been studied. We have previously extended the concept that GVHD is a purely $\mathrm{T}$ cell-mediated disease by showing that myeloid cells including neutrophils (15) and monocytes (16) contribute to GVHD development by tissue damage and MHC-II-dependent antigen presentation (17). Here we show that microglia, a prototypical tissue macrophage population in the CNS, contribute significantly to the pathogenesis of CNS-GVHD.

Our first observation that microglia cells exhibit an activated phenotype during GVHD with reduced numbers of branches is consistent with findings in CNS autoimmunity models describing this phenotype when experimental autoimmune encephalomyelitis (EAE) evolves (6). Our observation that CX3CR1 expression on microglia declined during GVHD is consistent with reports showing high CX3CR1 expression on resting microglia and a decline upon microglia activation (7). The morphological microglia changes occurred in parallel with the upregulation of MHC-II and CD80, which allow for antigen presentation to donor $\mathrm{T}$ cells and costimulation of these $\mathrm{T}$ cells, respectively. In agreement with this concept in CNS-GVHD, it was shown that non-hematopoietic antigen-presenting cells also contribute to peripheral GVHD, while classical dendritic cells were dispensable (18). Comparable to our findings in CNSGVHD, others have shown in EAE that myeloid antigen-presenting cells are recruited to the brain, which correlated with disease severity $(19,20)$, and that these cells present antigen to autoreactive $\mathrm{T}$ cells to mediate CNS inflammation (21).

In addition, we found increased levels of TNF in microglia during CNS-GVHD. TNF has direct cytotoxic effects on cells during GVHD (22), which could be responsible for the neuronal death observed previously after allo-HCT (2). Consistent with the concept that TNF plays a major role in CNS-GVHD, we found that $\mathrm{T}$ cell infiltration into the CNS and MHC-II expression on microglia were reduced when microglia cells were deficient in TNF.

We observed that TNF also increased in the syn-HCT group, although not to the same extent as in the allo-HCT group, which is likely due to the total-body irradiation-induced damage. The observation that $\mathrm{CD} 3^{+} \mathrm{T}$ cells were not increased in the syn-HCT groups indicates that a higher level of TNF may be required to attract $\mathrm{T}$ cells to the CNS, as was the case in the allo-HCT group.

Our finding that Th1 and Th17 cells declined in the CNS upon TAK1 inhibition is compatible with findings in other models of 
A
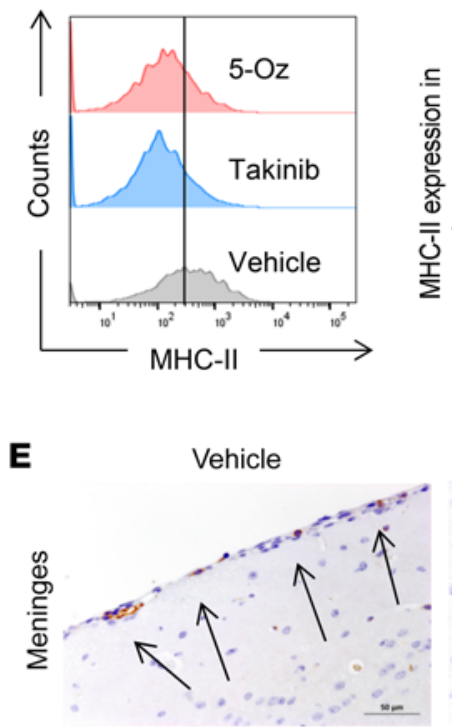

G

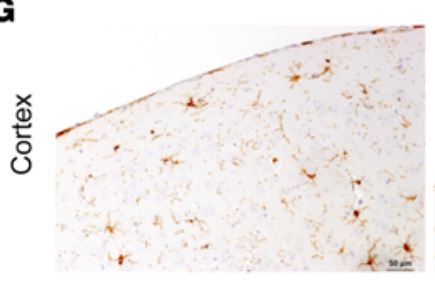

B

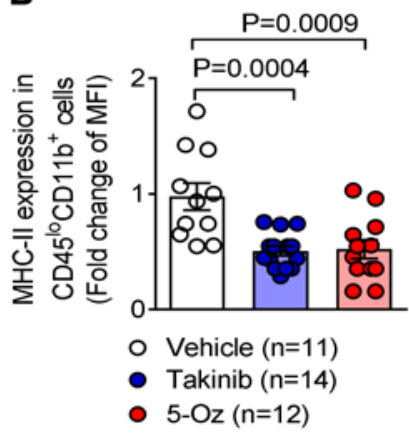

C

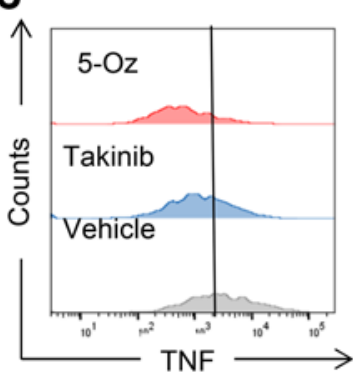

Takinib

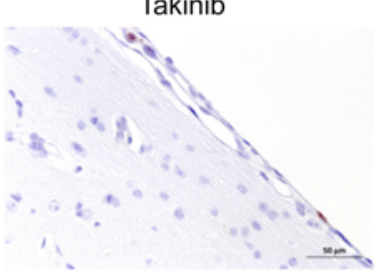

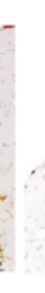
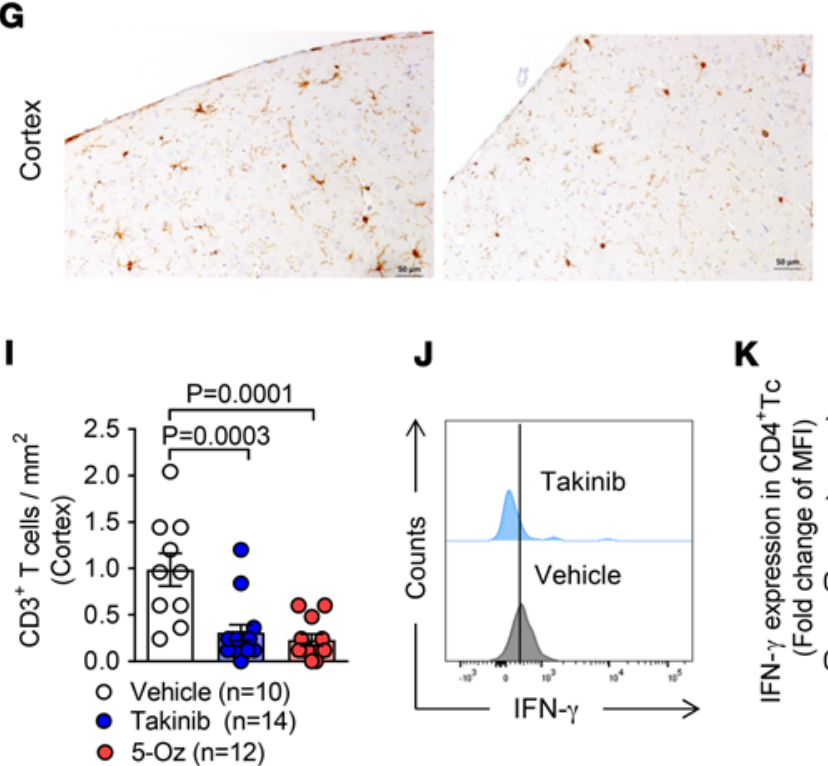

J

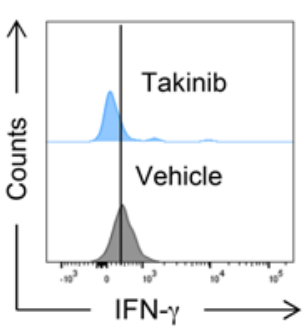

$\mathbf{K}$

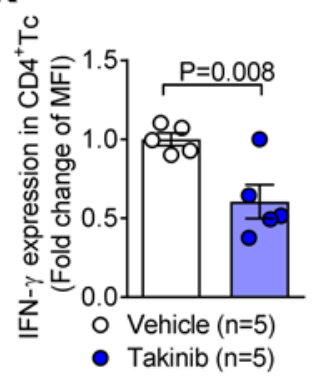

D



$\mathbf{F}$



H

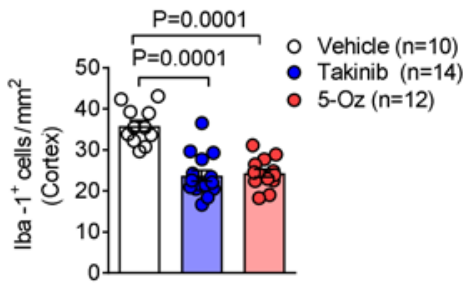

Iba- 1

L

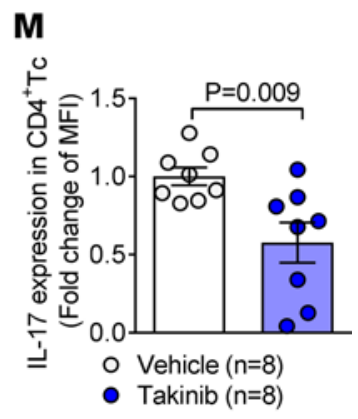

Figure 6. Therapeutic TAK1 inhibition alleviates CNS-CVHD-associated pathology and cognitive and memory deficits. (A-D) Flow cytometry plots and the respective scatter plots showing quantification (fold change of MFI) of MHC-II (A and $\mathbf{B}$ ) and TNF (C and $\mathbf{D}$ ) expression in microglia (CD45 ${ }^{\circ} \mathrm{CD} 11 \mathrm{~b}^{+}$) from brains of BALB/C mice treated with vehicle, takinib, or (5Z)-7-oxozeaenol (5-Oz) on day 14 after allo-HCT as indicated. The experiment was repeated 3 times, and the results (mean $\pm \mathrm{SEM}$ ) were pooled. $P$ values were calculated using 1-way ANOVA. (E-H) Histology of brain samples immunostained for CD ${ }^{+} \mathrm{T}$ cells and Iba- $1^{+}$cells from brains of BALB/c mice treated with vehicle $(n=10)$, takinib $(n=14)$, or 5-Oz $(n=12)$ on day 14 after allo-HCT as indicated. Representative images for meningeal $\mathrm{CD}^{+} \mathrm{T}$ cells $(\mathbf{E})$ and cortical Iba-1+ cells $(\mathbf{G})$ from each group are shown. Scale bars: $50 \mu \mathrm{m}$. The scatter plots show the number (per $\mathrm{mm}^{2}$ ) of meningeal $\mathrm{CD}^{+} \mathrm{T}$ cells $(\mathbf{F})$, cortical Iba- $1^{+}$cells $(\mathbf{H})$, and cortical CD3 ${ }^{+} \mathrm{T}$ cells $(\mathbf{I})$. The experiment was repeated 3 times, and the results (mean \pm SEM) were pooled. The $P$ values were calculated using 1-way ANOVA. ( and $\mathbf{K}$ ) Representative flow cytometry plots (J) and cumulative scatter plot (K) show quantification (fold change of MFI) of IFN- $\gamma$ expression in CD4+ $\mathrm{T}$ cells isolated on day 14 after allo-HCT from the CNS of BALB/c mice treated with vehicle $(n=5)$ or takinib $(n=5)$. (L and $\mathbf{M})$ Representative flow cytometry plots $(\mathbf{L})$ and cumulative scatter plot $(\mathbf{M})$ show quantification (fold change of MFI) of IL-17 expression in CD4 ${ }^{+}$T cells isolated on day 14 after allo-HCT from the CNS of BALB/c mice treated with vehicle $(n=8)$ or takinib $(n=8)$. (K and $\left.\mathbf{M}\right)$ The experiments were performed 3 times, and the results (mean \pm SEM) were pooled. $P$ values were calculated using 2-sided Student's unpaired $t$ test (K and $\mathbf{M}$ ).

CNS inflammation. Th1 and Th17 cells were shown to be proinflammatory and to promote the development of EAE $(23,24)$.

In our studies we also identify increased TAK1 activation and NF- $\mathrm{kB} / \mathrm{p} 38$ MAPK signaling activity in microglia during GVHD and show a functional role for TAK1, as selective Tak1 deletion in microglia reduced TNF production, $\mathrm{T}$ cell infiltrates in the CNS, and MHC-II levels. Mechanistically, the TAK1/
TNF axis was connected to VCAM-1 expression on endothelial cells. Genetic deletion of TNF or TAK1 inhibition reduced VCAM-1 expression during CNS-GVHD. T cell adhesion to endothelial cells is mediated via VCAM-1, which then allows T cells to exit the vessel and enter the CNS (11), supporting the concept that TAK1 inhibition reduced TNF and, subsequently, VCAM-1 expression, thereby decreasing donor $\mathrm{T}$ cell infiltra- 
A



E

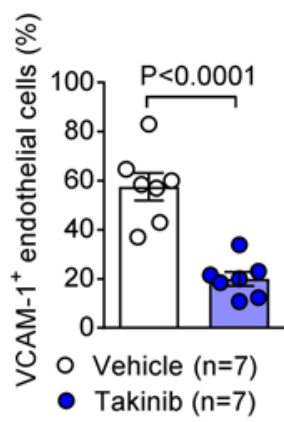

B



$\mathbf{F}$



C

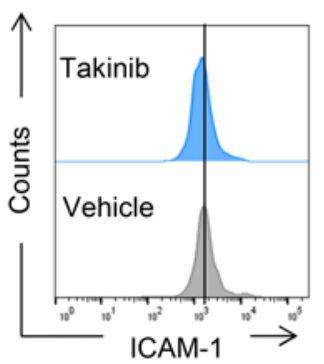

G $\operatorname{Tnf}^{n-1}$

D


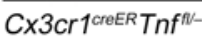


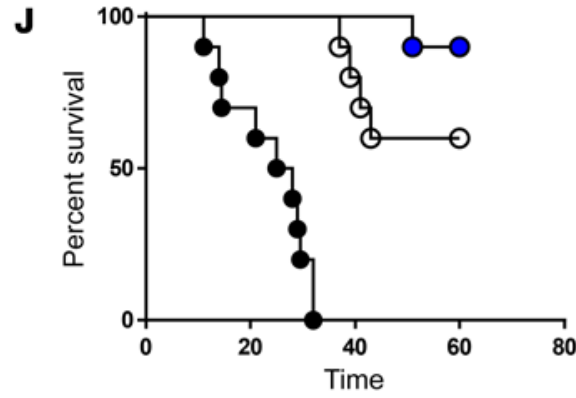

- $A M L(F L T 3-I T D / M L L-P T+B M(n=10)] \quad P<0.0001$

$\ominus$ AML (FLT3-ITD/MLL-PTD) + BM + Tc + Vehicle $(n=10)$

AML (FLT3-ITD/MLL-PTD) + BM + Tc + Takinib $(n=10)$


- $A M L(W E H I-3 B)+B M(n=10) \quad]^{P=0.002}$

$A M L(W E H I-3 B)+B M+$ TC $]$

+ Vehicle $(n=10)$
$P=0.01$

- AML (WEHI-3B) + BM + TC

+ Takinib $(n=10)$

Figure 7. VCAM-1 and ICAM-1 expression after allo-HCT declines upon TAK1 inhibition. (A-D) Representative flow cytometry plots and the respective cumulative scatter plots showing quantification of the fold change of MFI of VCAM-1 (A and $\mathbf{B}$ ) and ICAM-1 (C and $\mathbf{D})$ expression in endothelial cells (CD31+CD105 ${ }^{+}$) from the CNS of BALB/C mice treated with vehicle $(n=6)$ or takinib $(n=7)$ isolated on day 14 after allo-HCT. The experiment was performed once. (E-G) Immunofluorescence staining and scatter plots indicating the percentage of brain CD34+ endothelial cells expressing VCAM-1 and DAPI derived from BALB/c mice treated with vehicle $(n=7)$ or takinib $(n=7)(\mathbf{E})$ and from $\operatorname{Tnf}^{f / /}(n=7)$ or $\left[x 3 c r 7^{\text {creeR }} T n f^{f / /}(n=6)\right.$ mice $(\mathbf{F})$ on day 14 after allo-HCT. (G) Representative images from $T n f^{f /-}$ and $C \times 3 \mathrm{cr}^{\mathrm{free} R} \mathrm{Tnf} \mathrm{fl}^{f /-}$ mice, respectively, are shown. Scale bars: $50 \mu \mathrm{m}$. The experiment was performed once. (H) Scatter plot showing the percentage of open-arm entries by mice treated with vehicle $(n=11)$, takinib $(n=12)$, or $5-0 z(n=12)$ in an elevated plus maze test. (I) Scatter plot showing the percentage of time spent by mice treated with vehicle $(n=13)$, takinib $(n=12)$, or $5-0 z(n=12)$ in exploring a novel object in a novel object recognition test. The experiments were performed 3 times, and the results (mean \pm SEM) were pooled. (J) Survival rates of C57BL/6 mice with transplanted AML (FLT3-ITD/MLL-PTD) cells and BALB/C (WT) bone marrow (BM) along with (white and blue circles) and without (black circles) allogeneic T cells. (K) Survival rates of BALB/c mice with transplanted AML (WEHI-3B) cells and C57BL/6 BM (WT) along with (white and blue circles) and without (black circles) allogeneic T cells. The experiments were performed twice, and the results were pooled. $P$ values were calculated using 2-sided Student's unpaired $t$ test (B, D, and F), Mann-Whitney $U$ test (E), 1-way ANOVA ( $\mathbf{H}$ and $\mathbf{I}$ ), or 2-sided Mantel-Cox test (J and $\mathbf{K})$. 
A



C

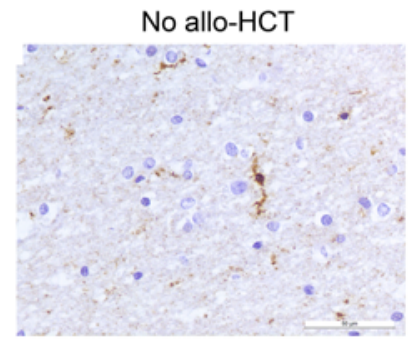

Allo-HCT/GVHD

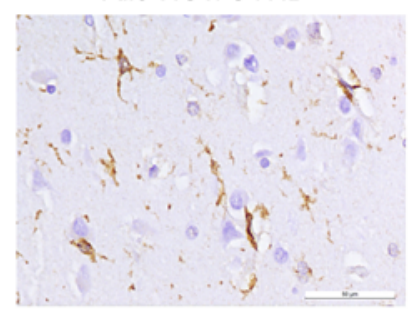

Allo-HCT/GVHD

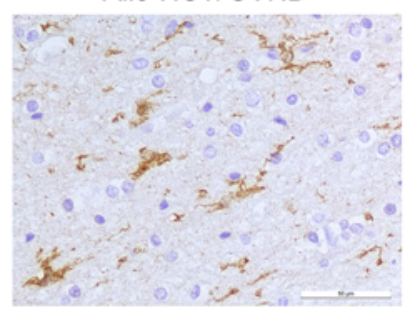

B Lobus frontalis (gray matter)

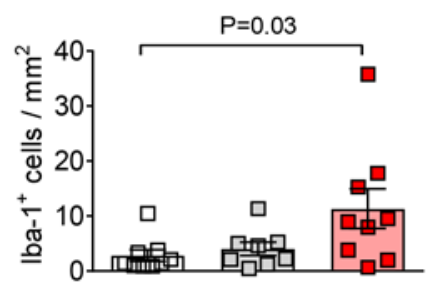

D Lobus frontalis (white matter)



口 No allo-HCT $(n=9)$

ㅁ Allo-HCT/no GVHD $(n=8$.

- Allo-HCT/GVHD $(n=9)$
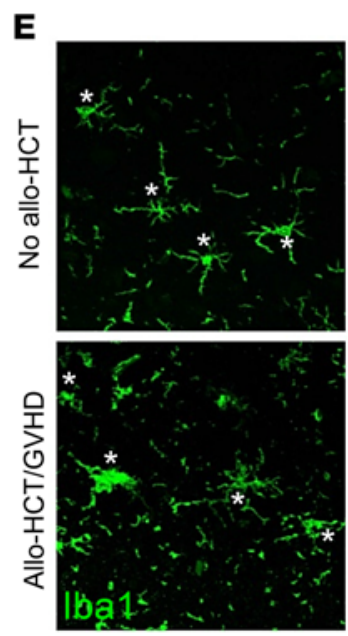

G

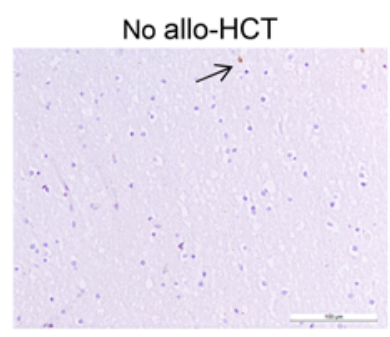

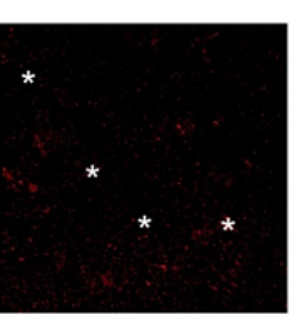
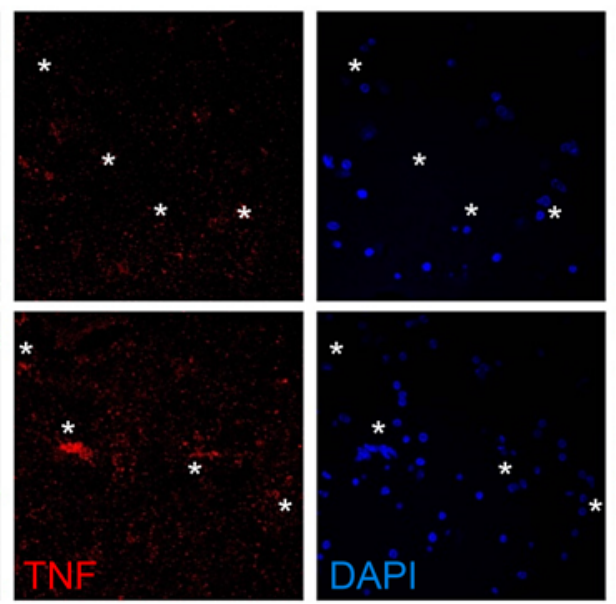
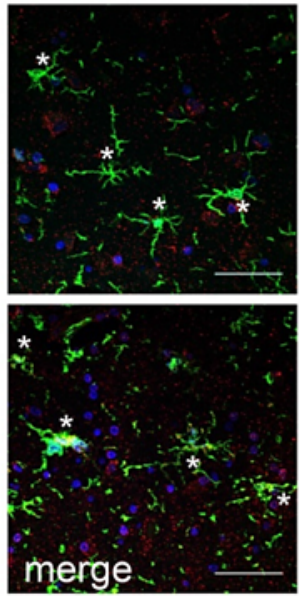

口 No allo-HCT $(n=9)$

口 Allo-HCT/no GVHD $(n=8)$

- Allo-HCT/GVHD $(n=9)$

$\mathbf{F}$



Allo-HCT/GVHD

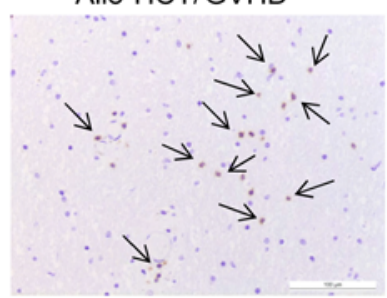

H Lobus frontalis



Figure 8. Microglia are activated and T cells infiltrate the CNS of GVHD patients. (A-D) Histology of brain samples immunostained for Iba-1+ ${ }^{+}$cells from the cortex of patients who had not undergone allo-HCT (No allo-HCT) $(n=9)$, had undergone allo-HCT with no GVHD symptoms (Allo-HCT/no GVHD) ( $n=8)$, or had undergone allo-HCT with grade III-IV GVHD symptoms (Allo-HCT/CVHD) $(n=9)$. (A and C) A representative image from each group is shown. Scale bars: $50 \mu \mathrm{m}$. (B and $\mathbf{D})$ The scatter plots show the number of $\mathrm{Iba}^{-1^{+}}$cells (per $\left.\mathrm{mm}^{2}\right)$ in gray matter (B) and white matter (D). The experiment was performed once. The $P$ values were calculated using 1-way ANOVA. (E and $\mathbf{F}$ ) Immunofluorescence staining of brain samples for Iba-1+ microglia, TNF, and DAPI from no allo-HCT $(n=8)$, allo-HCT/no GVHD $(n=9)$, and allo-HCT/GVHD $(n=9)$ groups of patients. (E) A representative image from no allo-HCT and allo-HCT/CVHD groups is shown. Scale bars: $50 \mu \mathrm{m}$. (F) Scatter plot indicating the percentage of Iba- $1^{+}$microglia expressing TNF from different groups. The experiment was repeated once. $P$ values were calculated using 1-way ANOVA. (G and H) Histology of brain samples immunostained for $\mathrm{CD}^{+} \mathrm{T}$ cells from the perivascular regions of brain from no allo-HCT $(n=9)$, allo-HCT/no GVHD $(n=8)$, and alloHCT/GVHD $(n=10)$ groups of patients. (G) Representative images from each group are shown. Scale bars: $50 \mu \mathrm{m}$. (H) The scatter plot shows the number of $\mathrm{CD3}^{+} \mathrm{T}$ cells (per $\mathrm{mm}^{2}$ ) in perivascular regions of brain. $P$ values were calculated using 1-way ANOVA. 
tion into the CNS. Consistent with a functional role for TAK1 in CNS-GVHD, the neurocognitive function of mice lacking TAK1 in microglia was improved. Besides the genetic Tak1 deletion approach, we also show that pharmacological TAK1 inhibition reduced TNF production and MHC-II expression by microglia cells and improved neurocognitive activity. Our previous work using Janus kinase inhibitors has shown the high potential of kinase inhibition to prevent or treat GVHD in mice (25) and patients (26). Our findings are potentially clinically relevant as treatment with the TAK1 inhibitor takinib did not affect GVL effects in 2 AML models. In agreement with the functional role of microglia-derived TNF for CNS-GVHD in mice, we observed an increase of the microglia cell number and their TNF production in the CNS of patients who developed GVHD.

In aggregate, we report a role for microglia in CNS-GVHD and identify the TAK1/TNF/MHC-II axis as a central mediator of this disease. Additionally, we provide a serine-threonine kinase inhibitor-based approach against GVHD-induced neurotoxicity identified based on RNA-Seq analysis of the CNS.

\section{Methods}

Mice. C57BL/6 (H-2Kb, Thy-1.2) and BALB/c (H-2Kd, Thy-1.2) mice were purchased either from Janvier Labs (France) or from the local stock of the animal facility at the University of Freiburg. Tak $1^{f / f l}$ and Cx3cr1 ${ }^{\text {creER }}$ Tak1 $1^{f l f l}$ (6), Cx3cr1 ${ }^{\text {creER }} R 26-y f p$ (6), CCR2 $2^{--}$(27), and $C \times 3 \mathrm{cr}^{\text {creER }} \mathrm{R} 26$-Tomato reporter mice were bred in the animal facility at the University of Freiburg. Tnfl-- and $C \times 3 \mathrm{crl}^{\mathrm{creER}} \mathrm{Tn} \mathrm{fl}^{\mathrm{l} / \text { - }}$ (27) mice were bred at the Weizmann Institute of Science, Rehovot, Israel. Mice were used between 6 and 14 weeks of age, and only female or male donor/ recipient pairs were used.

Bone marrow transplantation model and histopathology scoring. Bone marrow transplantation experiments were performed as previously described $(12,28)$. Briefly, recipients were exposed to lethal irradiation of 9-11 Gy. $\mathrm{CD}^{+}$and $\mathrm{CD}^{+} \mathrm{T}$ cells were isolated from donor spleens and enriched by positive selection with the MACS cell separation system (Miltenyi Biotec) according to the manufacturer's instructions. Anti-CD4 and anti-CD8 MicroBeads were used. Alternatively, $\mathrm{T}$ cells were isolated from donor spleens by negative selection (Pan $\mathrm{T}$ Cell Isolation Kit-II), with the MACS cell separation system (Miltenyi Biotec) according to the manufacturer's instructions. $\mathrm{CD} 4^{+} / \mathrm{CD} 8^{+} \mathrm{T}$ cell purity was at least $90 \%$ as assessed by flow cytometry (data not shown). To induce GVHD, $5 \times 10^{6}$ bone marrow cells and $\mathrm{CD}^{+} / \mathrm{CD}^{+}$ $\mathrm{T}$ cells from an MHC-mismatched donor (allogeneic stem cell transplantation) were given at a dosage of $3 \times 10^{5}$ (C57BL/6-derived into $\mathrm{BALB} / \mathrm{c})$ or $8 \times 10^{5}$ (BALB/c-derived into C57BL/6) i.v. on day 0. For behavioral studies, a sublethal dose of $5 \times 10^{5} \mathrm{BALB} / \mathrm{c}$-derived $\mathrm{T}$ cells was used. As a control, syngeneic stem cell transplantation (syn-HCT) was performed by injection of the same amount of bone marrow and $\mathrm{T}$ cells from a donor mouse with the same genetic background as that of the recipient. Slides of small intestine, large intestine, and liver specimens collected after allo-HCT were stained with H\&E and scored by an experienced pathologist blinded to the treatment groups. GVHD severity was determined according to a previously published histopathology scoring system (29).

RNA-Seq data were deposited in the NCBI's Gene Expression Omnibus database (GEO GSE141663). All antibodies are listed in Supplemental Table 7.
Statistics. For the sample size in the murine GVHD survival experiments, a power analysis was performed. A sample size of at least $n=$ 8 per group was determined by $80 \%$ power to reach a statistical significance of 0.05 to detect an effect size of at least 1.06. Differences in animal survival (Kaplan-Meier survival curves) were analyzed by Mantel-Cox test. The experiments were performed in a nonblinded fashion except for the GVHD severity scoring, the analysis of human tissue specimens, and behavioral experiments with the knockout mice. To obtain unbiased data, a pathologist blinded to both the genotype and the treatment group performed the histopathological scoring of GVHD severity. Only after finalization of the quantitative GVHD severity scores were the samples allocated to their genotypes/treatment group. Analysis of human brain specimens was also performed in a blinded manner, and the samples were allocated to the group after finalization of the analysis. All samples or mice were included in our analysis.

For statistical analysis of 2 groups, an unpaired 2-tailed Student's $t$ test was applied. All data were tested for normality applying the Kolmogorov-Smirnov test. If the data did not meet the criteria of normality, the Mann-Whitney $U$ test was applied. If more than 2 groups were analyzed, we used the Kruskal-Wallis test if nonparametric testing was suggested, and we performed a 1-way ANOVA in case of normally distributed data. Statistical analysis was performed using GraphPad Prism (GraphPad Software). Data are presented as mean and SEM (error bars). Differences were considered significant when the $P$ value was less than 0.05 .

Study approval. The study included formalin-fixed and paraffin-embedded (FFPE) tissue specimens of different brain regions from patients who died of causes unrelated to allo-HCT, patients who died after allo-HCT without GVHD, and patients who died after alloHCT and had GVHD (grade 3-4). The study was approved by the local ethics committee (protocol 547/14; Ethics Committee, University of Freiburg). Patients who underwent autopsy within a time period of 16 years (2001-2017) at the Institute of Neuropathology, Freiburg University Hospital, Germany, were analyzed. Patient characteristics are shown in Supplemental Tables 1-3.

A retrospective analysis of 503 patients undergoing allo-HCT at Freiburg University Medical Center with a follow-up of at least 12 months was performed. The analysis was approved by the Institutional Ethics Review Board of Freiburg University Medical Center (protocols 10024/13, 26/11, and 509/16). Written informed consent was obtained from each patient. All analyses of human data were carried out in compliance with the relevant ethical regulations. The analysis included neurological symptoms that occurred at the same time that a patient developed acute GVHD grade II-IV. Neurological symptoms related to infection or vascular events were not included. The observation time interval included the first 2 years after allo-HCT because acute GVHD occurs in $90 \%$ of all cases within this time interval. The multivariable logistic regression analysis included presence of acute GVHD grade II-IV, donor type (MRD, MUD, MMUD, haploidentical donor), conditioning (myeloablative versus reduced-intensity conditioning), sex, and age over 40 versus under 40 years as possible risk factors for the occurrence of neurological complications. Patient characteristics are provided in Supplemental Tables 5 and 6.

Animal protocols (protocols G-13/045, G13-116, G-15/018, G-17/063, X-13/07J, and X-15/10A) were approved by the Regierungspräsidium Freiburg (regional council), Freiburg, Germany (Federal 
Ministry for Nature, Environment and Consumers Protection). Animals at the Weizmann Institute of Science were handled according to protocols approved by the Weizmann Institute Animal Care Committee per international guidelines.

All other methods can be found in Supplemental Methods online; https://doi.org/10.1172/JCI130272DS1.

\section{Author contributions}

NRM and JMV performed the majority of the murine and cell line experiments and helped to develop the overall concept and write the manuscript. NRM and JMV are co-first authors. Their order in the author list was based on the first key experiments, which were performed and analyzed by NRM. PA, DE, and GI collected and analyzed patient samples. JSK performed cell sorting. SH, KH, NEK, DS, and TG helped with in vivo experiments. GA, MB, and LCM performed RNA sequencing and analyzed the data. TB helped to develop the concept and provided help with behavioral studies in mice. JD, JF, BRB, SJ, and MP contributed to critical analysis of the data, developed concepts, and provided reagents. RZ developed the overall concept, supervised the experiments, analyzed data, and wrote the manuscript.

\section{Acknowledgments}

We thank Eileen Barleon for performing immunohistochemistry, Klaus Geiger and Dieter Herchenbach for cell sorting, Heide Dierbach and Katja Graewe for tissue processing and staining, Britta Fritsch and Mate Dobrossy for providing the grip strength meter and elevated plus maze, respectively, Omar Mossad for his help with RNA isolation and endothelial isolation, and Takahiro Masuda for the inducible Cre-reporter line leakiness analysis. We acknowledge the team of the Genomics and Proteomics Core Facility, German Cancer Research Center (DKFZ), Heidelberg, Germany, for their sequencing service.

This study was supported by Deutsche Forschungsgemeinschaft (DFG) grants SFB TRR167 (to RZ, DE, TB, SJ, and MP), SFB1160 TP B09 (to RZ), and SFB 850 (to MB) and DFG individual grant 872/4-1 (to RZ); the European Union's GVHDCure (ERC consolidator grant to RZ); Deutsche Krebshilfe (70113473); the José Carreras Leukaemia Foundation (DJCLS 01R/2019 to RZ); Germany's Excellence Strategy (Centre for Integrative Biological Signalling Studies, EXC-2189, Project ID 390939984 to RZ); and the Interreg V European Regional Development Fund (European Union) program (project 3.2 TRIDIAG to RZ). PA was supported by the Else Kröner-Fresenius-Stiftung (EKFS 2015 A147). PA and DE are supported by the Berta-Ottenstein-Programme for Clinician Scientists. MB is supported by the German Federal Ministry of Education and Research (BMBF) within the framework of the e:Med research and funding concept (coNfirm, FKZ 01ZX1708F) and within the Medical Informatics Funding Scheme (MIRACUM, FKZ 01ZZ1606A-H).

Address correspondence to: Robert Zeiser, Department of Hematology, Oncology and Stem Cell Transplantation, University Medical Center Freiburg, Hugstetterstrasse 55, D-79106 Freiburg, Germany. Phone: 49.761.270.34580; Email: robert.zeiser@ uniklinik-freiburg.de.
1. Zeiser R, Blazar BR. Acute graft-versus-host disease - biologic process, prevention, and therapy. NEngl JMed. 2017;377(22):2167-2179.

2. Hartrampf $S$, et al. The central nervous system is a target of acute graft versus host disease in mice. Blood. 2013;121(10):1906-1910.

3. Kaliyaperumal S, et al. CD8-predominant T-cell CNS infiltration accompanies GVHD in primates and is improved with immunoprophylaxis. Blood. 2014;123(12):1967-1969.

4. Unger ER, Sung JH, Manivel JC, Chenggis ML, Blazar BR, Krivit W. Male donor-derived cells in the brains of female sex-mismatched bone marrow transplant recipients: a Y-chromosome specific in situ hybridization study. J Neuropathol Exp Neurol. 1993;52(5):460-470.

5. Shortt J, Hutton E, Faragher M, Spencer A. Central nervous system graft-versus-host disease post allogeneic stem cell transplant. Br J Haematol. 2006;132(2):245-247.

6. Goldmann T, et al. A new type of microglia gene targeting shows TAK1 to be pivotal in CNS autoimmune inflammation. Nat Neurosci. 2013;16(11):1618-1626.

7. Cho SH, et al. CX3CR1 protein signaling modulates microglial activation and protects against plaque-independent cognitive deficits in a mouse model of Alzheimer disease. J Biol Chem. 2011;286(37):32713-32722.

8. Sethi G, Ahn KS, Pandey MK, Aggarwal BB. Celastrol, a novel triterpene, potentiates TNF- induced apoptosis and suppresses invasion of tumor cells by inhibiting NF-kB-regulated gene products and TAK1-mediated NF- $\kappa \mathrm{B}$ activation. Blood. 2007;109(7):2727-2735.

9. Adhikari A, Xu M, Chen ZJ. Ubiquitin-mediated activation of TAK1 and IKK. Oncogene. 2007;26(22):3214-3226.

10. Sakurai H, Miyoshi H, Toriumi W, Sugita T. Functional interactions of transforming growth factor $\beta$-activated kinase 1 with IkappaB kinases to stimulate NF- $\mathrm{B}$ activation. J Biol Chem. 1999;274(15):10641-10648.

11. Shimizu Y, et al. Four molecular pathways of T cell adhesion to endothelial cells: roles of LFA-1, VCAM-1, and ELAM-1 and changes in pathway hierarchy under different activation conditions. J Cell Biol. 1991;113(5):1203-1212.

12. Mathew NR, et al. Sorafenib promotes graft-versus-leukemia activity in mice and humans through IL-15 production in FLT3-ITD-mutant leukemia cells. Nat Med. 2018;24(3):282-291.

13. Balaguer-Rosello A, et al. Noninfectious neurologic complications after allogeneic hematopoietic stem cell transplantation. Biol Blood Marrow Transplant. 2019;25(9):1818-1824.

14. Saad AG, Alyea EP, Wen PY, Degirolami U, Kesari S. Graft-versus-host disease of the CNS after allogeneic bone marrow transplantation. J Clin Oncol. 2009;27(30):e147-e149.

15. Schwab L, et al. Neutrophil granulocytes recruited upon translocation of intestinal bacteria enhance graft-versus-host disease via tissue damage. Nat Med. 2014;20(6):648-654.

16. Klämbt V, et al. A novel function for $\mathrm{P} 2 \mathrm{Y} 2$ in myeloid recipient-derived cells during graft-versushost disease. JImmunol. 2015;195(12):5795-5804.

17. Hülsdünker J, et al. Neutrophils provide cellular communication between ileum and mesenteric lymph nodes at graft-versus-host disease onset. Blood. 2018;131(16):1858-1869.

18. Koyama M, et al. Recipient nonhematopoietic antigen-presenting cells are sufficient to induce lethal acute graft-versus-host disease. Nat Med. 2011;18(1):135-142.

19. Sagar D, Lamontagne A, Foss CA, Khan ZK, Pomper MG, Jain P. Dendritic cell CNS recruitment correlates with disease severity in EAE via CCL2 chemotaxis at the blood-brain barrier through paracellular transmigration and ERK activation. J Neuroinflammation. 2012;9:245.

20. Jordão MJC, et al. Single-cell profiling identifies myeloid cell subsets with distinct fates during neuroinflammation. Science. 2019;363(6425):eaat7554.

21. Greter M, et al. Dendritic cells permit immune invasion of the CNS in an animal model of multiple sclerosis. Nat Med. 2005;11(3):328-334.

22. Hill GR, et al. Differential roles of IL- 1 and TNF- $\alpha$ on graft-versus-host disease and graft versus leukemia. J Clin Invest. 1999;104(4):459-467.

23. Komiyama Y, et al. IL-17 plays an important role in the development of experimental 
autoimmune encephalomyelitis. J Immunol. 2006;177(1):566-573.

24. Jäger A, Dardalhon V, Sobel RA, Bettelli E, Kuchroo VK. Th1, Th17, and Th9 effector cells induce experimental autoimmune encephalomyelitis with different pathological phenotypes. J Immunol. 2009;183(11):7169-7177.

25. Spoerl S, et al. Activity of therapeutic JAK $1 / 2$ blockade in graft-versus-host disease. Blood. 2014;123(24):3832-3842.

26. Zeiser R, et al. Ruxolitinib in corticosteroidrefractory graft-versus-host disease after allogeneic stem cell transplantation: a multicenter survey. Leukemia. 2015;29(10):2062-2068.

27. Wolf Y, et al. Autonomous TNF is critical for in vivo monocyte survival in steady state and inflammation. JExp Med. 2017;214(4):905-917.

28. Wilhelm K, et al. Graft-versus-host disease is enhanced by extracellular ATP activating P2X7R. Nat Med.2010;16(12):1434-1438.

29. Kaplan DH, Anderson BE, McNiff JM, Jain D, Shlomchik MJ, Shlomchik WD. Target antigens determine graft-versus-host disease phenotype. JImmunol. 2004;173(9):5467-5475. 